$$
\begin{aligned}
& \alpha_{0} \\
& \lrcorner-1 \\
& \text { AVgebra \& } \\
& \text { Number } \\
& \text { 列 }
\end{aligned}
$$

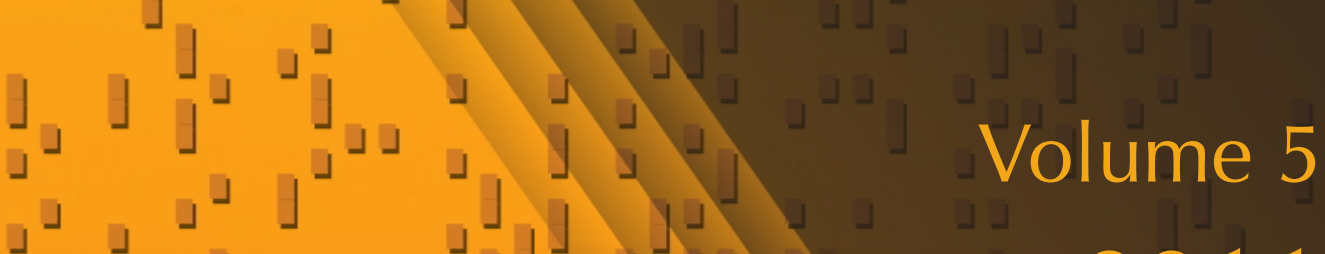

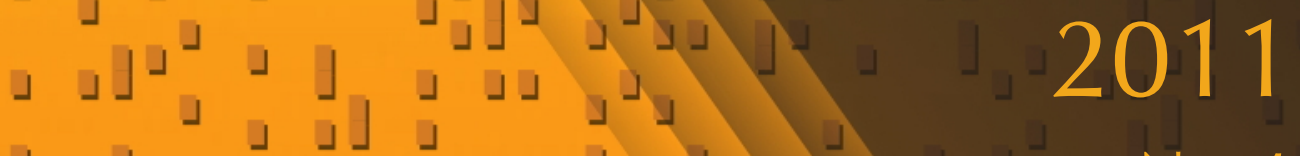

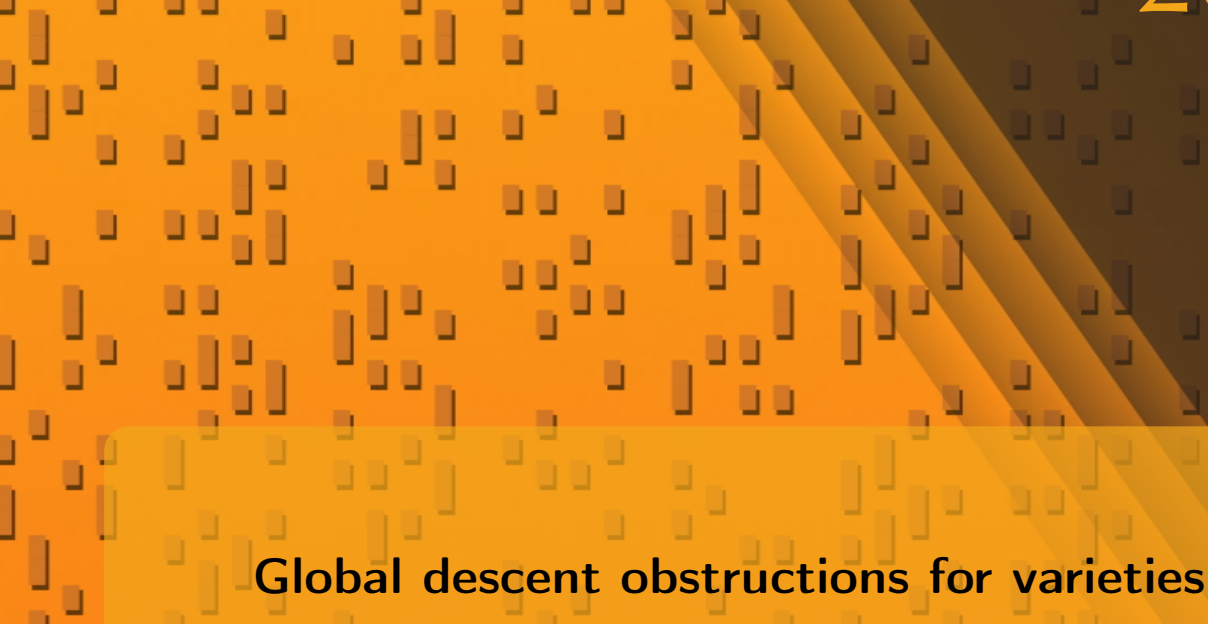

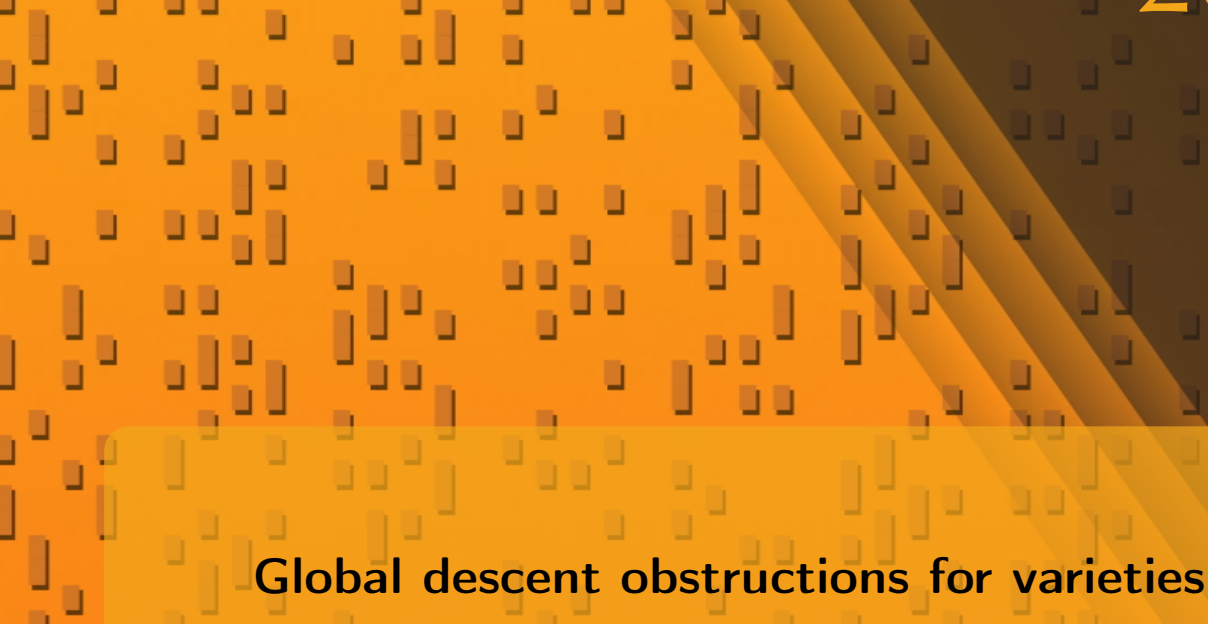

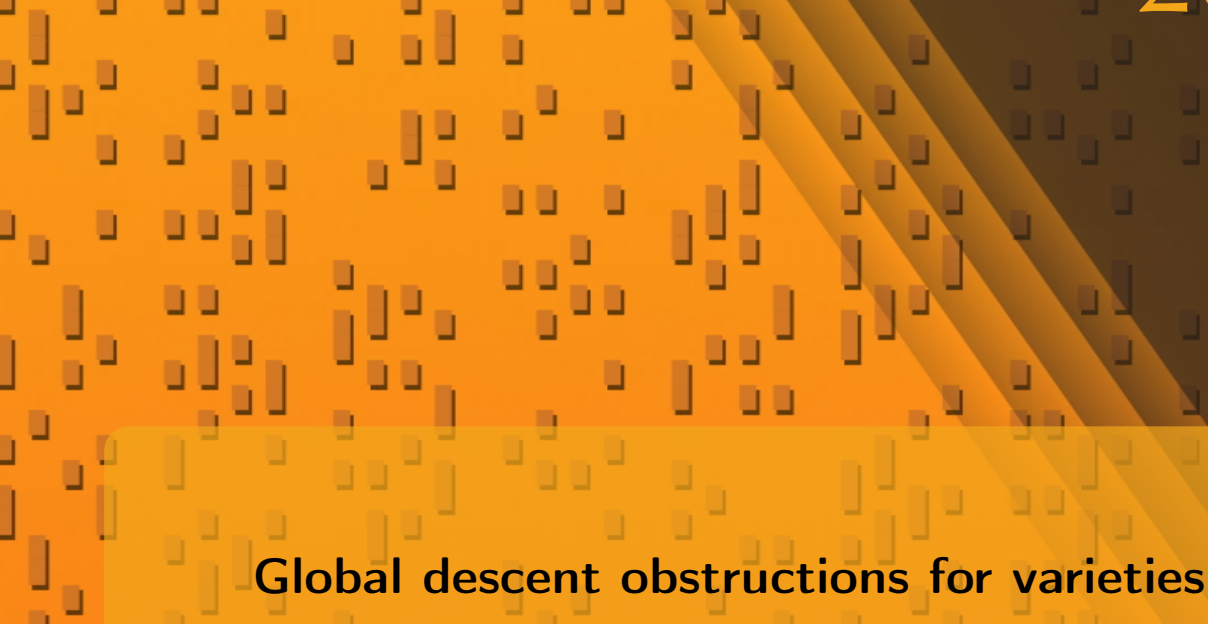

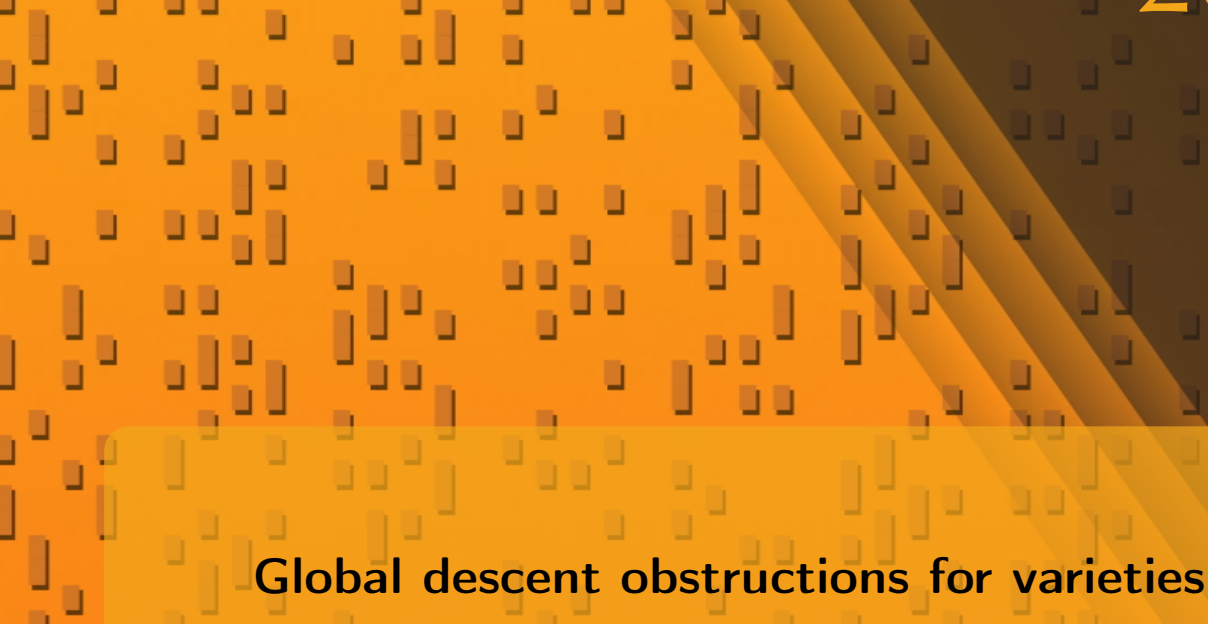

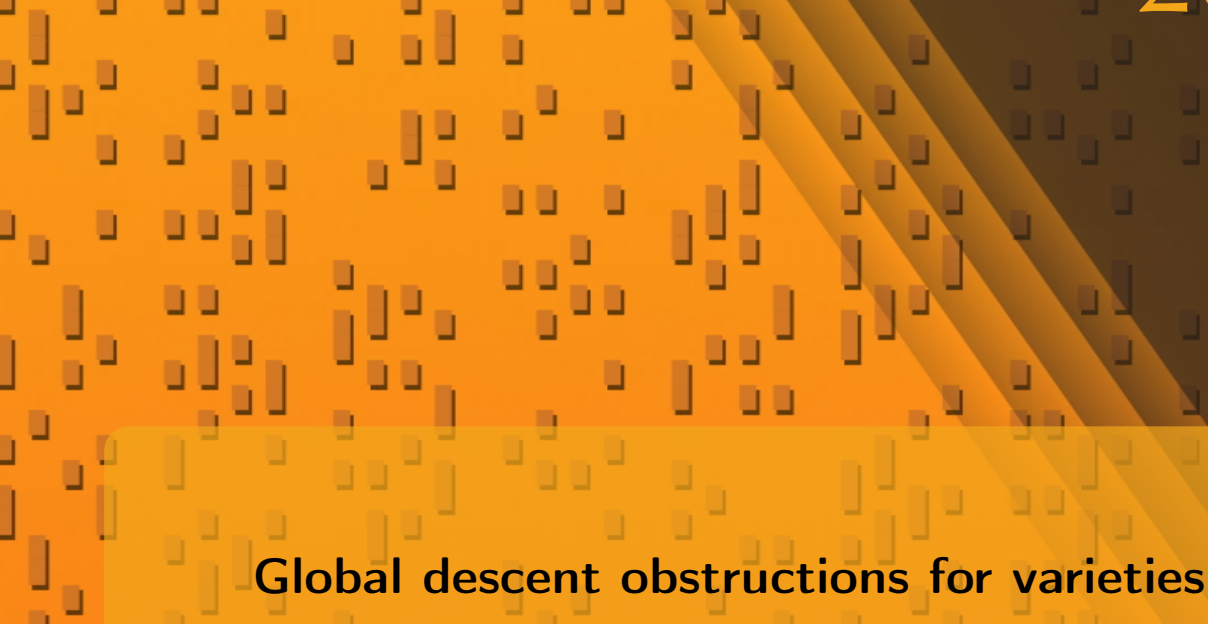

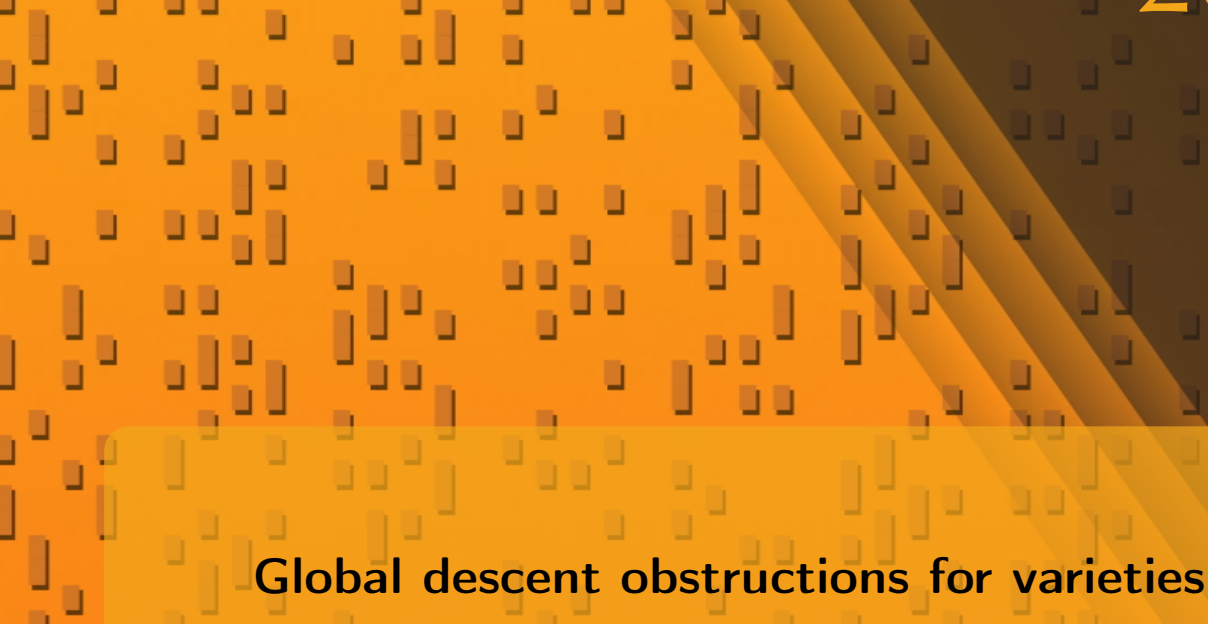

$$
\begin{aligned}
& \text { No. } 4
\end{aligned}
$$

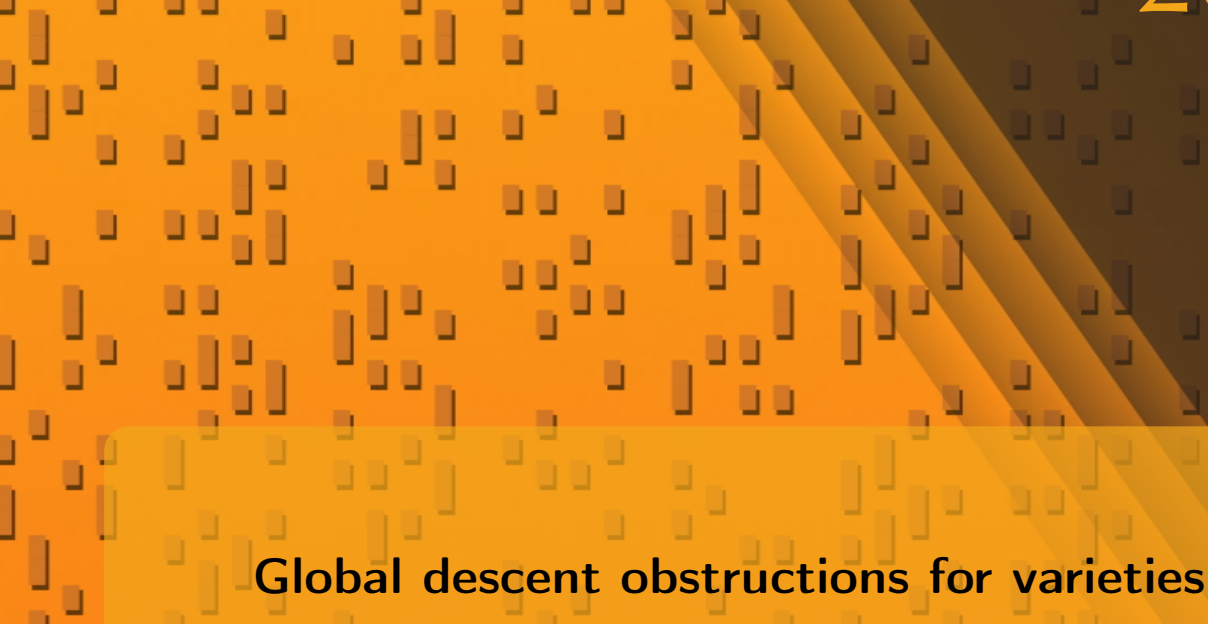

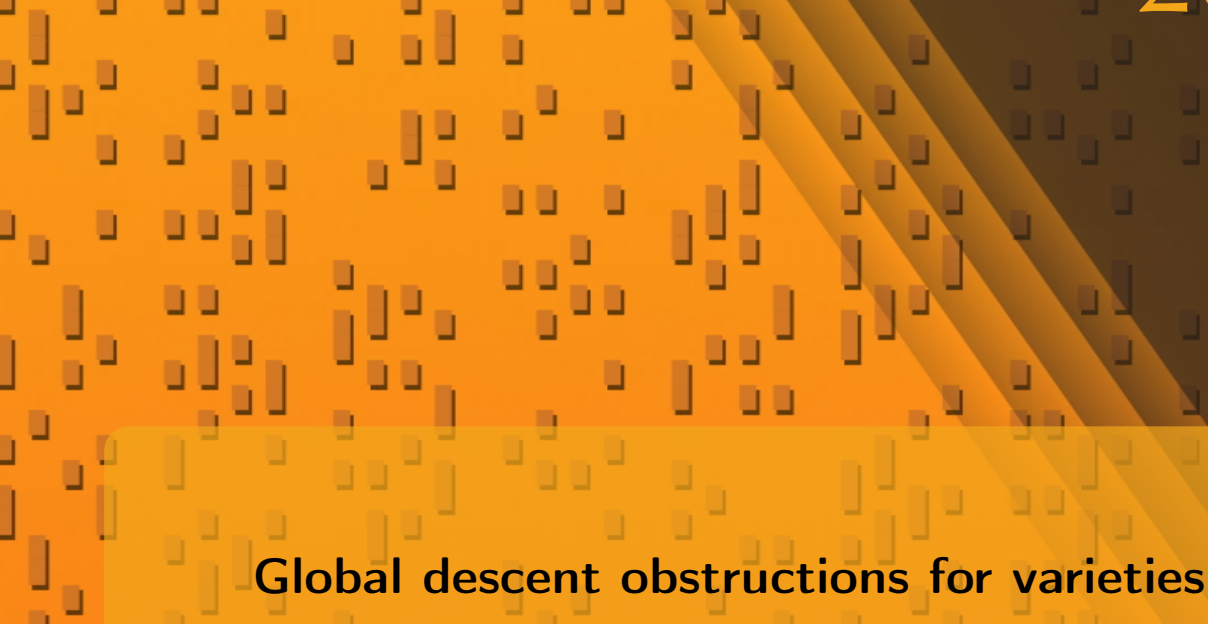

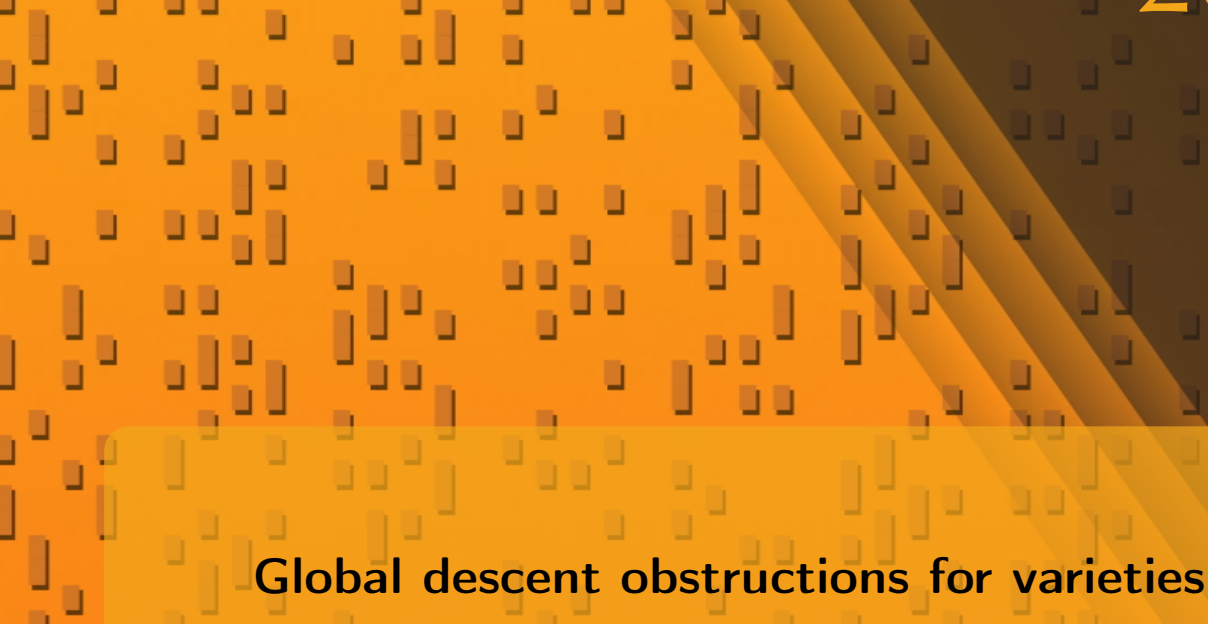

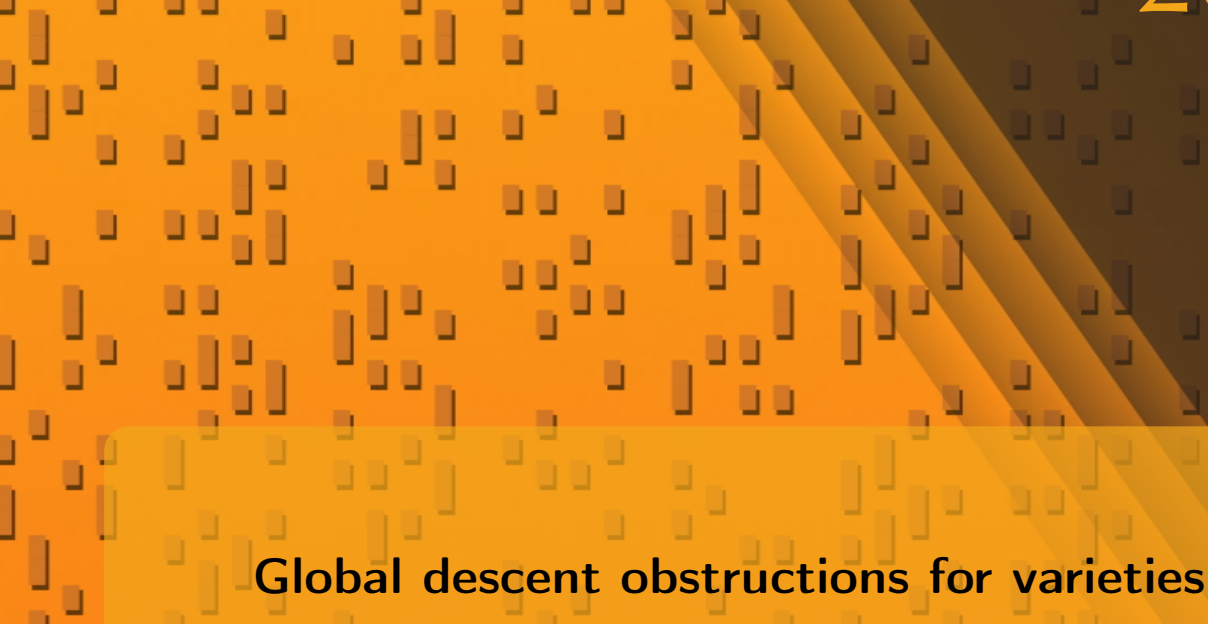

Jean-Marc Couveignes and Emmanuel Hallouin

\lrcorner

\title{
Global descent obstructions for varieties
}

」 


\title{
Global descent obstructions for varieties
}

\author{
Jean-Marc Couveignes and Emmanuel Hallouin
}

\begin{abstract}
We show how to transport descent obstructions from the category of covers to the category of varieties. We deduce examples of curves having $\mathbf{Q}$ as field of moduli, that admit models over every completion of $\mathbf{Q}$, but have no model over $\mathbf{Q}$.
\end{abstract}

\section{Introduction}

If $k$ is a field, a $k$-variety is by definition a separated scheme of finite type over $\operatorname{Spec}(k)$. A $k$-curve is a variety of dimension 1 over $k$. A $k$-surface is a variety of dimension 2 over $k$.

1a. Statement of the main results. This work constructs descent obstructions in the category of varieties. For example, we prove the following:

Theorem 1.1. There exists a projective, integral and smooth curve over $\overline{\mathbf{Q}}$, having $\mathbf{Q}$ as field of moduli, which has models over all the completions of $\mathbf{Q}$ but not over $\mathbf{Q}$ itself.

The main idea is to start from a descent obstruction in the category of finite morphisms (also called covers) of curves, and to transport it into various other categories: the category of quasiprojective surfaces, the category of proper surfaces, and finally the category of smooth curves.

Theorem 1.2. Let $k$ be a field of characteristic zero, and $k^{a}$ be an algebraic closure of $k$. Let $X_{k}$ be a smooth, projective, geometrically integral curve over $k$ and let $X$ denote the base change to $k^{a}$ of $X_{k}$. Let $Y$ be a smooth, projective, integral curve over $k^{a}$ and let $\varphi: Y \rightarrow X$ be a (possibly ramified) cover over $k^{a}$, having $k$ as field of moduli. There exists a smooth, projective, integral curve over $k^{a}$ having $k$ as field of moduli and having exactly the same fields of definition as the initial cover $\varphi$.

This work is supported by the French Agence Nationale pour la Recherche through project ALGOL (ANR-07-BLAN-0248).

MSC2000: primary 11R34; secondary 12G05, 11G35, 14D22.

Keywords: algebraic curve, global field, descent, field of definition, field of moduli, stack. 
Descent obstructions have been constructed in the categories of $G$-covers and covers [Coombes and Harbater 1985; Dèbes and Fried 1994; Couveignes and Granboulan 1994] and in the category of dynamical systems [Silverman 1995]. As far as we know, no example of purely global descent obstruction was known for varieties. Mestre [1991] gave some examples of local obstructions for hyperelliptic curves. Dèbes and Emsalem [1999] give a criterion in order to have a model over its field of moduli for a curve. This criterion involves a particular model for the quotient of the curve by its automorphism group. Dèbes and Emsalem also prove that the local-global principle applies to the descent problem for a curve together with its automorphisms. However they leave open the question of the local-global principle for a curve (or a variety in general). Purely global descent obstructions for covers have been constructed by Ros and Couveignes:

Theorem 1.3 [Couveignes and Ros 2004, Corollaire 2]. There exists a connected ramified $\overline{\mathbf{Q}}$-cover of $\mathbf{P}_{\mathbf{Q}}^{1}$ having $\mathbf{Q}$ as field of moduli, having models over all the completions of $\mathbf{Q}$, but having no model over $\mathbf{Q}$.

Applying Theorem 1.2 to these obstructions, one proves Theorem 1.1.

1b. Overview of the paper. Let $k$ be a field with characteristic zero. Let $X_{k}$ be a smooth, projective and geometrically integral curve over $k$ and set $X=X_{k} \times{ }_{k} k^{a}$ to be the pullback of $X_{k}$ along the inclusion $k \hookrightarrow k^{a}$. Our starting point is a smooth projective and integral curve $Y$ over $k^{a}$ and a nonconstant morphism $\varphi: Y \rightarrow X$. We look for a variety having the same field of moduli and the same fields of definition as $\varphi$. A natural candidate is the complement $X \times Y-G(\varphi)$ of the graph $G(\varphi)$ of $\varphi$ in the product $X \times Y$. We call it the mark of $\varphi$. We expect this surface to have the same field of moduli and the same fields of definition as $\varphi$. In order to prove it, we construct a morphism from the stack of models of $\varphi$ onto the stack of models of the mark of $\varphi$. Then, we try to prove that this morphism is an equivalence of categories.

In Section 2, we recall the definition of the stack of "models" of an algebraic variety over $k^{a}$ (or of a cover of curves over $k^{a}$ ). Then, we explain how a morphism between the two gerbes associated with two objects relates the definition and moduli properties of either objects. It turns out that the key point is to control the group of automorphisms of the two objects. We first prove, in Section 3, that the base curve $X$ of our starting cover $\varphi$ can be assumed to have no nontrivial $k^{a}$-automorphism. In other words, we construct another $k$-curve $X_{k}^{\prime}$ without any nontrivial $k^{a}$-automorphism and a $k^{a}$-cover $Y^{\prime} \rightarrow X_{k}^{\prime} \times{ }_{k} k^{a}$ having the same field of moduli and the same fields of definition as $\varphi$. In Section 4 , we take it for granted that $X$ has no nontrivial automorphism and we prove that the mark of $\varphi$ has the same field of moduli and the same fields of definition as $\varphi$. In Section 5, we assume 
that the field of moduli of the cover $\varphi$ is $k$ and we construct a proper normal $k^{a}$ surface having $k$ as field of moduli and the same fields of definition as $\varphi$. This proper surface is a cover of $X \times Y$, strongly ramified along the graph of $\varphi$. Finally, in Section 6, we construct a projective $k^{a}$-curve, having $k$ as field of moduli, and having the same fields of definition as the initial cover $\varphi$. This curve lies on the previous surface, and is obtained by deformation of a stable curve having the same automorphism group as the surface.

Notation. If $k$ is a field, we denote by $k^{a}$ its algebraic closure. Let $l$ be a $k$ extension and let $X_{l}$ be an $l$-variety. We denote by $\operatorname{Aut}_{l}\left(X_{l}\right)$ or simply $\operatorname{Aut}\left(X_{l}\right)$ the group of automorphisms of the $l$-variety $X_{l}$ (that is, automorphisms over $\operatorname{Spec}(l)$ ). We denote by $\operatorname{Aut}_{k}\left(X_{l}\right)$ the group of automorphisms of the $k$-scheme $X_{l}$ (that is, automorphisms over $\operatorname{Spec}(k))$. For $f \in l\left(X_{l}\right)$ we write $(f)_{0}$ for the divisor of zeros of $f$, and $(f)_{\infty}$ for the divisor of poles.

\section{Stack of "models"}

In this section $k$ is a field of characteristic zero and $k^{a}$ is an algebraic closure of $k$.

2a. The conjugate of a variety. Let $X$ be a $k^{a}$-variety. We denote by $\pi: X \rightarrow$ $\operatorname{Spec}\left(k^{a}\right)$ the structural morphism. Let $\sigma: k^{a} \rightarrow k^{a}$ be a $k$-isomorphism. We denote by ${ }^{\sigma} X$ the $k^{a}$-variety defined to be $X$ itself with the structural morphism $\sigma_{\pi}=\operatorname{Spec}(\sigma)^{(-1)} \circ \pi$. The square below is cartesian and ${ }^{\sigma} \pi$ is the pullback of $\pi$ along $\operatorname{Spec}(\sigma)$.

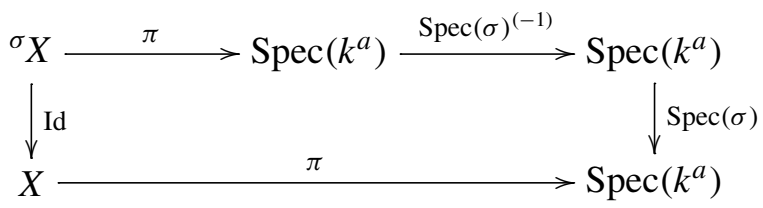

With this (slightly abusive) notation one has ${ }^{\tau}\left({ }^{\sigma}(\pi)\right)={ }^{\tau \sigma} \pi$ and ${ }^{\tau}\left({ }^{\sigma}(X)\right)={ }^{\tau \sigma} X$. If $X$ is an affine variety, then ${ }^{\sigma} X$ is obtained from $X$ by letting $\sigma$ act on the coefficients in the defining equations of $X$.

2b. The field of moduli. It is natural to ask whether $X$ and ${ }^{\sigma} X$ are isomorphic. They are certainly isomorphic as schemes (and even equal by definition), but as varieties over $k^{a}$, they are isomorphic if and only if there exists an isomorphism $\phi_{\sigma}$ that makes the following diagram commute:

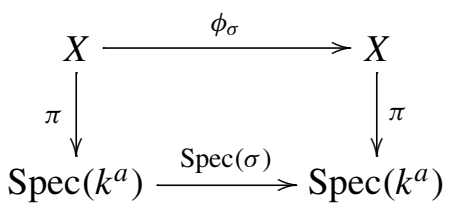


The existence of such a square means that the isomorphism $\operatorname{Spec}(\sigma)$ of $\operatorname{Spec}\left(k^{a}\right)$ lifts to an isomorphism $\phi_{\sigma}$ of $X$. The set of such $\sigma$ is a subgroup of $\operatorname{Gal}\left(k^{a} / k\right)$. The fixed field of this subgroup is a finite extension of $k$ called the field of moduli of $X$.

2c. Fields of definition. Another natural question: given $l \subset k^{a}$ an algebraic extension of $k$, do an $l$-variety $\pi_{l}: X_{l} \rightarrow \operatorname{Spec}(l)$ and a cartesian square

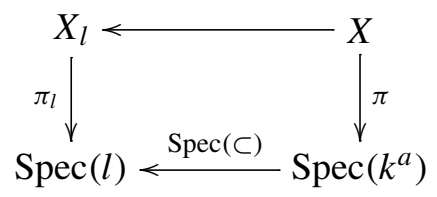

(where the line below is the spectrum of the inclusion) exist or not? If such a square exists we say that $l$ is a field of definition of $X$. We say that $\pi_{l}: X_{l} \rightarrow \operatorname{Spec}(l)$ is a model of $\pi: X \rightarrow \operatorname{Spec}\left(k^{a}\right)$ over $l$. One may wonder whether it is important to impose the arrow below in the definition above. The answer is yes, it is, in general. The existence of such a cartesian square may depend on the chosen arrow below. However, if $k$ is the field of moduli of $X$, then we may compose the cartesian squares in (1) and (2) as

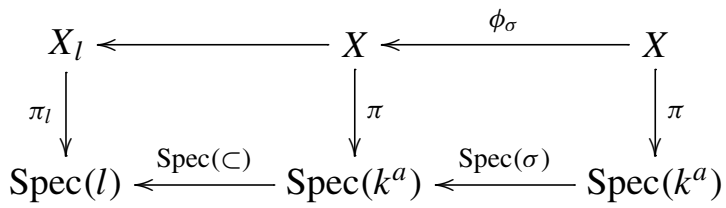

and choose any of the arrows below.

Another simple observation: if $X$ has a model $\pi_{k}: X_{k} \rightarrow \operatorname{Spec}(k)$ over $k$, then $k$ is the field of moduli of $X$. Indeed, we write $X$ as a fiber product $X=X_{k} \times{ }_{k} k^{a}$ and we take for $\phi_{\sigma}$ the fiber product $\operatorname{Id}_{X_{k}} \times_{\operatorname{Spec}(k)} \operatorname{Spec}(\sigma)$ where $\operatorname{Id}_{X_{k}}: X_{k} \rightarrow X_{k}$ is the identity on $X_{k}$. One may wonder whether the converse is true or not.

2d. Descent obstructions. Let's assume that $k$ is the field of moduli of the $k^{a}$ variety $X$. Does there exist a model over $k$ ? If the answer is no, we say that there is a descent obstruction. In case $k$ is a number field, we say that the obstruction is purely global if $k$ is the field of moduli and if there is no model over $k$, but for every place $v$ of $k$ there exists a model of $X$ over some extension $l \subset k^{a}$ such that $l$ can be embedded in the completion $k_{v}$ of $k$ at $v$.

2e. The fibered category of "models" of a variety. We denote by $\mathrm{Et} / \operatorname{Spec}(k)$ the category of finite étale morphisms over $\operatorname{Spec}(k)$. An object $U$ in this category is a structural morphism $\operatorname{Spec}(l) \rightarrow \operatorname{Spec}(k)$, where $k \hookrightarrow l$ is a finite étale $k$-algebra. We define a covering of $U$ to be a surjective family $\left(U_{i} \rightarrow U\right)_{i}$ of morphisms in 
$\mathrm{Et} / \operatorname{Spec}(k)$. This turns $\mathrm{Et} / \operatorname{Spec}(k)$ into a site called the étale site on $\operatorname{Spec}(k)$. It satisfies the three axioms of a site: the pullback of a covering exists and is a covering, a covering of a covering is a covering, and the identity is a covering. ${ }^{1}$

Given a $k^{a}$-variety $X$, we define the fibered category over Et / Spec $(k)$ of its "models". For any $k$-algebra $l$, we must say what we mean by a "model" of $X$ over $\operatorname{Spec}(l)$. If $l$ is a field, we say that an $l$-variety $\pi_{l}: X_{l} \rightarrow \operatorname{Spec}(l)$ is a "model" of $X$ over $\operatorname{Spec}(l)$ if and only if there exist an embedding $e: l \hookrightarrow k^{a}$ over $k$ and a cartesian square

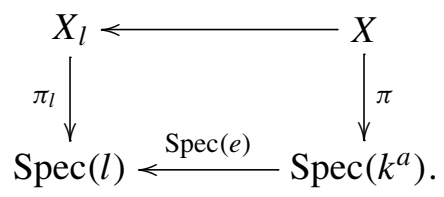

This time, we insist on the fact that we do not fix an embedding of $l$ into $k^{a}$. In particular, if $l$ is a subfield of $k^{a}$ containing $k$, we accept models of $X$ but also models of all its conjugates. So the word model here is less restrictive than in Section 2c. That is why we write the word model between quotation marks in that case. As we have already noticed, the two notions coincide when $k$ is the field of moduli of $X$. If $l$ is any finite étale algebra over $k$, then it is a direct product of finitely many finite field extensions of $k$. We define a "model" of $X$ over $\operatorname{Spec}(l)$ to be a disjoint union of "models" of $X$ over every connected component of $\operatorname{Spec}(l)$.

Definition 2.1 (the category $\mathbb{M}_{X}$ of "models" of $X$ ). Let $X$ be a $k^{a}$-variety. The category of "models" of $X$, denoted $\mathbb{M}_{X}$, is the category whose objects are all "models" of $X$ over all finite étale $k$-algebras, and whose morphisms are the cartesian squares

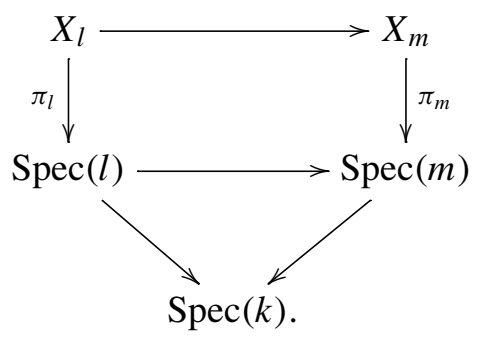

The functor that associates $\operatorname{Spec}(l)$ to every "model" over $\operatorname{Spec}(l)$ turns $\mathbb{M}_{X}$ into a fibered category over $\operatorname{Et} / \operatorname{Spec}(k)$; we denote by $\mathbb{M}_{X}(l)$ or $\mathbb{M}_{X}(\operatorname{Spec}(l))$ the fiber over $\operatorname{Spec}(l)$.

\footnotetext{
${ }^{1}$ Note that in this paper, we use the word covering in the context of sites. We keep the word cover for a finite morphism between two varieties, for example, two smooth projective and geometrically integral curves.
} 
In particular, we can pull back a "model" $X_{l} \rightarrow \operatorname{Spec}(l)$ along any morphism $\operatorname{Spec}(m) \rightarrow \operatorname{Spec}(l)$ over $\operatorname{Spec}(k)$. Let's note that pulling back is not quite innocent since it can turn a model into its conjugates, so to say.

2f. Descent data. We recall that under mild conditions, the fibered category $\mathbb{M}_{X}$ is a stack. We need a few definitions and elementary results about descent data (see Giraud [1964] or Vistoli's notes [2005]). Let $\mathbb{S}$ be a site and let $\mathbb{X}$ be a fibered category over $\mathbb{S}$. Let $U$ be an object in $\mathbb{S}$ and let $U=\left(U_{i} \rightarrow U\right)_{i}$ be a covering of $U$. A descent datum from $U$ to $U$ is a collection of objects $X_{i} \rightarrow U_{i}$. For every $i$ and every $j$, we also want an isomorphism $\phi_{i j}: \pi_{2}^{*}\left(X_{j}\right) \rightarrow \pi_{1}^{*}\left(X_{i}\right)$, where $\pi_{1}$ and $\pi_{2}$ are the two "projections" in the cartesian diagram

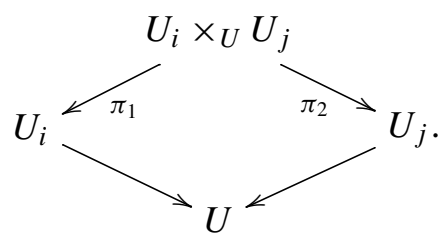

We also require the compatibility relation

$$
\pi_{12}^{*}\left(\phi_{i j}\right) \circ \pi_{23}^{*}\left(\phi_{j k}\right)=\pi_{13}^{*}\left(\phi_{i k}\right) \text { for any } i, j \text {, and } k \text {. }
$$

Here the $\pi_{12}, \pi_{23}, \pi_{31}$ are the partial "projections" in the cube

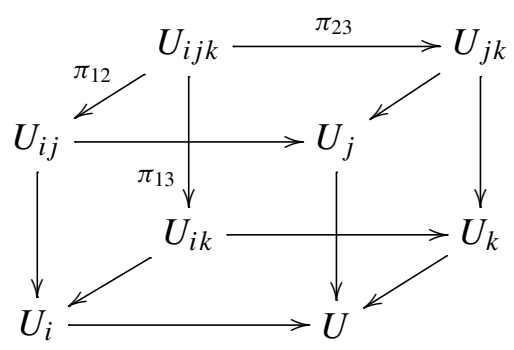

and $U_{i j}=U_{i} \times_{U} U_{j}$ and $U_{i j k}=U_{i} \times{ }_{U} U_{j} \times{ }_{U} U_{k}$.

A morphism of descent data is a collection of local morphisms that are compatible with the glueing morphisms on either sides. Thus we obtain a category $\operatorname{Desc}_{\mathbb{X}}(U, U)$ for every covering $U$ of $U$. We denote by $\mathbb{X}(U)$ the fiber of $\mathbb{X}$ above $U$. There is a functor $\mathbb{X}(U) \rightarrow \operatorname{Desc}_{\mathbb{X}}(U, U)$ that associates to any object over $U$ the collection of its restrictions over the $U_{i}$. These constructions are functorial. For example, if $\mathbb{Y}$ is another fibered category and $\mathbb{F}: \mathbb{X} \rightarrow \mathbb{Y}$ a cartesian functor, then $\mathbb{F}$ induces a functor from $\mathbb{X}(U)$ to $\mathbb{Y}(U)$ and a functor from $\operatorname{Desc}_{\mathbb{X}}(\mathcal{U}, U)$ to $\operatorname{Desc} \mathbb{Y}(U, U)$. Also, the composite functors $\mathbb{X}(U) \rightarrow \mathbb{Y}(U) \rightarrow \operatorname{Desc} \mathbb{Y}(\mathcal{U}, U)$ and $\mathbb{X}(U) \rightarrow \operatorname{Desc}_{\mathbb{X}}(\mathcal{U}, U) \rightarrow \operatorname{Desc}_{\mathbb{Y}}(\mathcal{U}, U)$ are isomorphic. A fibered category 
$\mathbb{X}$ over a site $\mathbb{S}$ is a stack if and only if all the functors $\mathbb{X}(U) \rightarrow \operatorname{Desc}_{\mathbb{X}}(U, U)$ are equivalences of categories.

2g. When $\mathbb{M}_{X}$ is a stack, respectively a gerbe. If $X$ is a $k^{a}$-variety, then $\mathbb{M}_{X}$ is a fibered category over $\mathrm{Et} / \operatorname{Spec}(k)$ and it makes sense to ask if it is a stack or not. We first notice that if $l \subset m$ are two finite field extensions of $k$, then $\operatorname{Spec}(m) \rightarrow$ $\operatorname{Spec}(l)$ is a covering of $\operatorname{Spec}(l)$. If further $m / l$ is Galois, then a descent datum from $\operatorname{Spec}(m)$ to $\operatorname{Spec}(l)$ is a model $\pi_{m}: X_{m} \rightarrow \operatorname{Spec}(m)$ of $X$ over $\operatorname{Spec}(m)$ and, for every $\sigma$ in $\operatorname{Gal}(m / l)$, an automorphism $\phi_{\sigma}: X_{m} \rightarrow X_{m}$ of $l$-scheme, such that the diagram

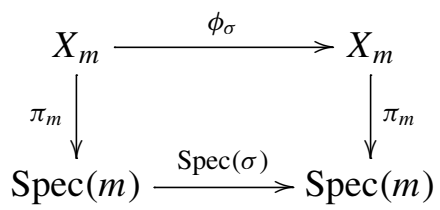

commutes. We stress that the $\phi_{\sigma}$ need not be automorphisms of the $m$-variety $X_{m}$ but only automorphisms of the $l$-scheme $X_{m}$. Let $\operatorname{Aut}_{l}\left(X_{m}\right)$ denote the set of automorphisms of the $l$-scheme $X_{m}$. The meaning of condition (3) is that the map $\operatorname{Spec}(\sigma) \mapsto \phi_{\sigma}$ must be a group homomorphism from $\operatorname{Aut}_{\operatorname{Spec}(l)}(\operatorname{Spec}(m))$ into $\operatorname{Aut}_{l}\left(X_{m}\right)$.

Proposition 2.2. Let $X$ be a variety over $k^{a}$. If $X$ is affine or projective or if every finite subset of $X\left(k^{a}\right)$ is contained in an affine subvariety, then the fibered category $\mathbb{M}_{X}$ is a stack over $\operatorname{Et} / \operatorname{Spec}(k)$.

Proof. This is a consequence of Weil's descent theory. See the initial article of Weil [1956] or Serre's book [1959, Chapter V, Section 4].

Let us recall that a locally nonempty and locally connected stack is called a gerbe. More precisely, a stack $\mathbb{X}$ over a site $\mathbb{S}$ is a gerbe if and only if

(1) for every object $U$ in $\mathbb{S}$, there exists a covering $\left(U_{i} \rightarrow U\right)_{i}$ of $U$ such that the fibers over the $U_{i}$ are nonempty,

(2) given two objects $X \mapsto U$ and $Y \mapsto U$ above $U$, there exists a covering $\left(U_{i} \rightarrow U\right)_{i}$ such that for every $i$ the pullbacks $X \times_{U} U_{i}$ and $Y \times_{U} U_{i}$ are isomorphic over $U_{i}$, and

(3) for every object $U$ in $\mathbb{S}$, the fiber $\mathbb{X}(U)$ is a groupoid.

The stack $\mathbb{M}_{X}$ of "models" of a variety $X$ always satisfies conditions one and three, whereas the second holds true if and only if $k$ is the field of moduli of $X$.

2h. The stack, next the gerbe of "models" of a cover of curves. Since the starting point of our construction is a cover of curves, we now define the stack of "models" of a cover of curves. Let $X_{k}$ be a smooth, projective, geometrically integral curve 
over $k$. We set $X=X_{k} \times_{k} k^{a}$. Let $Y$ be a smooth projective and integral curve over $k^{a}$ and let $\varphi: Y \rightarrow X$ be a nonconstant morphism. We say that $\varphi$ is a cover of $X$. An isomorphism between two covers $\varphi: Y \rightarrow X$ and $\psi: Z \rightarrow X$ is an isomorphism of $k^{a}$-varieties $i: Y \rightarrow Z$ such that $\psi \circ i=\varphi$.

The conjugate of a cover. If $\sigma$ is a $k$-automorphism of $k^{a}$, the conjugate variety ${ }^{\sigma} X$ is obtained from $X$ by composing the structural morphism on the left with $\operatorname{Spec}(\sigma)^{(-1)}$. The same is true for $Y$. So any $k^{a}$-morphism $\varphi$ from $Y$ to $X$ can be seen as a $k^{a}$-morphism ${ }^{\sigma} \varphi$ from ${ }^{\sigma} Y$ to ${ }^{\sigma} X$. Since $X$ is the fiber product of $X_{k}$ and $\operatorname{Spec}\left(k^{a}\right)$ over $\operatorname{Spec}(k)$, we have a canonical isomorphism $\phi_{\sigma}=\operatorname{Id}_{X_{k}} \times \operatorname{Spec}(k)$ $\operatorname{Spec}(\sigma)$ between $X$ and ${ }^{\sigma} X$. The composite map $\phi_{\sigma}^{(-1)} \circ^{\sigma} \varphi$ is a morphism of $k^{a}-$ varieties from ${ }^{\sigma} Y$ to $X$. We call it the conjugate of $\varphi$ by $\sigma$. We may denote it ${ }^{\sigma} \varphi$ also by abuse of notation.

The field of moduli. This is the fixed field by the subgroup of $\operatorname{Gal}\left(k^{a} / k\right)$ consisting of all $\sigma$ such that ${ }^{\sigma} \varphi$ is isomorphic to $\varphi$.

Fields of definition, models. If $l \subset k^{a}$ is an algebraic extension of $k$, we set $X_{l}=$ $X_{k} \times_{k} l$. Let $Y_{l}$ be a smooth projective and geometrically connected $l$-curve. Let $\varphi_{l}: Y_{l} \rightarrow X_{l}$ be a cover. If we lift $\varphi_{l}$ along the spectrum of the inclusion $l \subset k^{a}$, we obtain a morphism from $Y_{l} \times_{l} k^{a}$ onto $X=X_{l} \times_{l} k^{a}$. If this cover is isomorphic to $\varphi: Y \rightarrow X$, we say that $\varphi_{l}$ is a model of $\varphi$ over $l$. So it makes sense to ask whether (purely global) obstructions to descent for covers of curves exist or not. It is proved in [Couveignes and Ros 2004] that such obstructions do exist.

The fibered category of "models" of a cover. Given a finite étale $k$-algebra $l$, we explain the meaning of a "model" of $\varphi$ over $l$. If $l$ is a finite field extension of $k$, we set $X_{l}=X_{k} \times_{k} l$. Let $Y_{l}$ be a smooth projective and geometrically integral curve over $\operatorname{Spec}(l)$, and let $\varphi_{l}: Y_{l} \rightarrow X_{l}$ be a cover defined over $\operatorname{Spec}(l)$. We pick now any embedding $e: l \rightarrow k^{a}$ over $k$. The pullback of $X_{l}$ along $\operatorname{Spec}(e)$ is $X$ (up to unique isomorphism) and we have the following diagram:

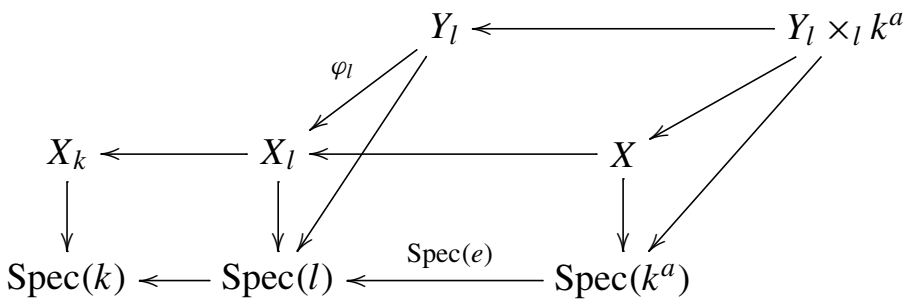

We say that $\varphi_{l}$ is a "model" of $\varphi$ if the cover $\varphi_{l} \times \operatorname{Spec}(l) \operatorname{Spec}\left(k^{a}\right): Y_{l} \times_{l} k^{a} \rightarrow X$ is isomorphic to $\varphi$. Again the choice of the embedding $e$ does not matter. We just ask such an embedding to exist. If $l$ is any finite étale algebra over $k$, we define a "model" of $\varphi$ over $\operatorname{Spec}(l)$ to be a disjoint union of "models" of $\varphi$ over every 
connected component of $\operatorname{Spec}(l)$. We write $\mathbb{M}_{\varphi}$ for the category of all models of $\varphi$. This is a fibered category over $\operatorname{Et} / \operatorname{Spec}(k)$. The following proposition is a consequence of Weil's descent theorem.

Proposition 2.3. Let $X_{k}$ be a smooth, projective, geometrically integral curve over $k$ and set $X=X_{k} \times_{k} k^{a}$. Let $Y$ be a smooth projective and integral curve over $k^{a}$ and let $\varphi: Y \rightarrow X$ be a nonconstant morphism of $k^{a}$ curves. Then the fibered category $\mathbb{M}_{\varphi}$ is a stack over $\operatorname{Et} / \operatorname{Spec}(k)$.

As in the case of varieties, the stack $\mathbb{M}_{f}$ is a gerbe if and only if $k$ is the field of moduli of $f$.

2i. Transporting obstructions. The next result summarizes the previous sections.

Proposition 2.4. Let $X$ be a $k^{a}$-variety (or a cover of curves). Then $k$ is the field of moduli of $X$ if and only if the stack $\mathbb{M}_{X}$ is a gerbe. The field $l$ is a field of definition of $X$ if and only if the fiber $\mathbb{M}_{X}(l)$ is not empty.

Let $X$ and $Y$ be two $k^{a}$-varieties. A cartesian morphism of stacks $\mathbb{F}: \mathbb{M}_{X} \rightarrow \mathbb{M}_{Y}$ is a functor of fibered categories (it transforms cartesian squares into cartesian squares). Such an $\mathbb{F}$ associates an $l$-model $\mathbb{F}\left(X_{l}\right)$ of $Y$ to every $l$-model $X_{l}$ of $X$, and commutes with base change. One can use it to relate descent obstruction for $X$ and for $Y$.

Proposition 2.5. Let $X$ and $Y$ be either $k^{a}$-varieties or covers of curves. Suppose that there exists a morphism $\mathbb{F}: \mathbb{M}_{X} \rightarrow \mathbb{M}_{Y}$ of stacks.

(1) If $k$ is the field of moduli of $X$, then $k$ is the field of moduli of $Y$.

(2) If $l$ is a field of definition of $X$, then $l$ is also a field of definition of $Y$.

(3) Assume further that $k$ is the field of moduli of $X$ and that $\mathbb{F}$ is fully faithful.

Then $l$ is a field of definition of $X$ if and only if $l$ is a field of definition of $Y$.

Proof. Assertions 1 and 2 result from Proposition 2.4 and Section 2g. Assertion 3 is a consequence of the following lemma.

Lemma 2.6. Let $\mathbb{X}$ and $\mathbb{Y}$ be two gerbes over a site $\mathbb{S}$ and let $\mathbb{F}: \mathbb{X} \rightarrow \mathbb{Y}$ be a cartesian morphism. If $\mathbb{E}$ is fully faithful, then $\mathbb{E}$ is essentially surjective.

Proof. Let $U$ be an object in $\mathbb{S}$ and let $Y \rightarrow U$ be an object in the fiber $\mathbb{Y}(U)$. Locally $\mathbb{X}(U)$ is not empty: there exist a covering $\left(U_{i} \rightarrow U\right)_{i}$ of $U$ and objects $X_{i} \in$ $\mathbb{X}\left(U_{i}\right)$ for every $i$. Set $Y_{i}=Y \times_{U} U_{i}$. Locally, $Y_{i}$ and $\mathbb{F}\left(X_{i}\right)$ are isomorphic: there exists a covering $\left(U_{i j} \rightarrow U_{i}\right)_{j}$ such that $Y_{i} \times_{U_{i}} U_{i j}$ and $\mathbb{F}\left(X_{i} \times_{U_{i}} U_{i j}\right)$ are isomorphic. Set $X_{i j}=X_{i} \times_{U_{i}} U_{i j}$ and $Y_{i j}=Y_{i} \times_{U_{i}} U_{i j}$.

The set of objects $\left(Y_{i j} \rightarrow U_{i j}\right)_{i j}$ defines a descent datum from $\left(U_{i j} \rightarrow U\right)_{i j}$ to $U$; indeed for every $i, j, i^{\prime}, j^{\prime}$, pulling back identity gives rise to isomorphisms

$$
\Phi_{i j i^{\prime} j^{\prime}}: Y_{i^{\prime} j^{\prime}} \times_{U_{i^{\prime} j^{\prime}}} U_{i j i^{\prime} j^{\prime}} \rightarrow Y_{i j} \times_{U_{i j}} U_{i j i^{\prime} j^{\prime}}
$$


which clearly satisfy the compatibility conditions (3) of Section 2 f. Since $\mathbb{F}$ is fully faithful, there exist isomorphisms

$$
\Psi_{i j i^{\prime} j^{\prime}}: X_{i^{\prime} j^{\prime}} \times_{U_{i^{\prime} j^{\prime}}} U_{i j i^{\prime} j^{\prime}} \rightarrow X_{i j} \times_{U_{i j}} U_{i j i^{\prime} j^{\prime}}
$$

which, in turn, satisfy the compatibility conditions (3) of Section $2 \mathrm{f}$. We deduce that there exists $X \rightarrow U$ in $\mathbb{X}(U)$ such that $\mathbb{F}(X)=Y$.

We now give an example of morphism between two stacks of "models" of a variety.

Proposition 2.7. Let $X$ be a integral variety over $k^{a}$ having $k$ as field of moduli and let $G$ be a finite subgroup of $\operatorname{Aut}_{k^{a}}(X)$ which is normal in the group $\operatorname{Aut}_{k}(X)$. Assume that every orbit of $G$ is contained in an affine open subset of $X$. Then there is a morphism from $\mathbb{M}_{X}$ to $\mathbb{M}_{X / G}$, where $X / G$ denotes the quotient variety of $X$ by $G$.

Let $Y \subset X / G$ be the complement of the branch locus of $X \rightarrow X / G$. This is an open subvariety of $X / G$ and there is a morphism of stacks from $\mathbb{M}_{X}$ to $\mathbb{M}_{Y}$.

Proof. We first need to define the image of an object. Let $l$ be an extension of $k$ and let $X_{l}$ be a "model" of $X$ in $\mathbb{M}_{X}(l)$. The elements of $G$ may not be all defined over $l$, but there exists a finite Galois extension $m$ of $l$ over which they are. Set $X_{m}=X_{l} \times_{l} m$. Let $p_{m}: X_{m} \rightarrow X_{m} / G$ be the canonical projection onto the quotient of $X_{m}$ by the group $G$. According to Section $2 \mathrm{~g}$, there exists a group homomorphism $\sigma \mapsto \phi_{\sigma} \operatorname{from} \operatorname{Gal}(m / l)$ to $\operatorname{Aut}_{l}\left(X_{m}\right)$. Since $G$ is a normal subgroup of $\operatorname{Aut}_{k}(X)$, it is normal in $\operatorname{Aut}_{l}\left(X_{m}\right)$ also. Thus for every $g \in G$ and every $\sigma \in \operatorname{Gal}(m / l)$, one has $\phi_{\sigma} \circ g \circ \phi_{\sigma}^{-1} \in G$. We deduce that $p_{m} \circ \phi_{\sigma} \circ g=p_{m} \circ \phi_{\sigma}$ for every $g \in G$. This implies that $\phi_{\sigma}$ factorizes into $\psi_{\sigma}: X_{m} / G \rightarrow X_{m} / G$. By uniqueness of this factorization, the correspondence $\sigma \mapsto \psi_{\sigma}$ is a group homomorphism from $\operatorname{Gal}(m / l)$ to $\operatorname{Aut}_{l}\left(X_{m} / G\right)$; therefore the quotient $X_{m} / G$ descends to $l$.

Next, we define the image of a morphism. Let $X_{i} \rightarrow \operatorname{Spec}\left(l_{i}\right)$ for $i=1,2$ be two "models" of $X$. One can complete a cartesian square involving the $X_{i}$ as

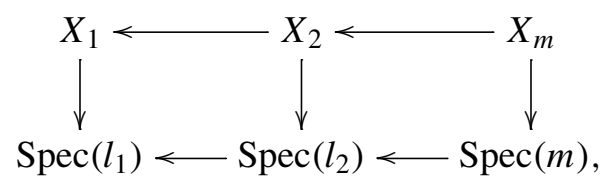

where $m$ is a finite Galois extension of $k$ such that all the elements of $G$ are defined over $m$. We know that there exist isomorphisms $\Phi_{1}$ and $\Phi_{2}$ making the following 
diagrams commute:
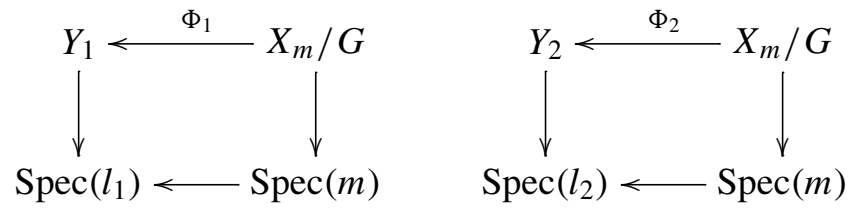

The image of the starting cartesian square is nothing but

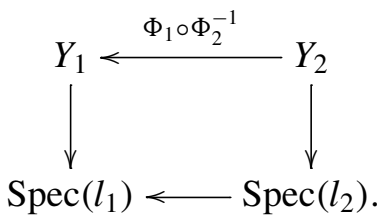

This completes the proof of the first statement. The second is true since taking the branch locus commutes with base changes.

\section{Cancellation of the automorphism group of the base curve}

In this section, $k$ is a field of characteristic zero, $k^{a}$ is an algebraic closure of it, and $l \subset k^{a}$ is an algebraic extension of $k$. Let $X_{k}$ be a projective, smooth, geometrically integral curve over $k$ and set $X=X_{k} \times{ }_{k} k^{a}$. We assume that we are given a smooth projective and integral curve $Y$ over $k^{a}$ and a cover $\varphi: Y \rightarrow X$ having $k$ as field of moduli. We want to construct covers having the same field of moduli and the same fields of definition as $\varphi$ but satisfying additional properties. For example, we would like the base curve $X$ to have no nontrivial $k^{a}$-automorphism. We first prove that the degree of the cover can be multiplied by any prime integer not dividing the initial degree.

Proposition 3.1. Let $X_{k}$ be a smooth, projective, geometrically integral curve over $k$ and set $X=X_{k} \times{ }_{k} k^{a}$. Let $Y$ be a smooth projective and integral curve over $k^{a}$ and let $\varphi: Y \rightarrow X$ be a degree d cover. For every prime $p$ not dividing $d$, there exist a smooth projective curve $Y^{\prime}$ over $k^{a}$ and a degree pd cover $\psi: Y^{\prime} \rightarrow X$ having the same field of moduli and the same fields of definition as $\varphi$.

Proof. Let $f \in k\left(X_{k}\right)$ be a nonconstant function whose divisor is simple and does not meet the ramification locus of $\varphi$. The equation $h^{p}=f$ defines a degree $p$ extension of $k\left(X_{k}\right)$. We denote by $X_{k}^{\prime}$ the smooth, projective, geometrically integral curve corresponding to this function field and we set $X^{\prime}=X_{k}^{\prime} \times_{k} k^{a}$. The morphism $v: X^{\prime} \rightarrow X$ is a degree $p$ cyclic cover. We fix an algebraic closure $\Omega$ of $k^{a}(X)$ and embeddings of $k^{a}\left(X^{\prime}\right)$ and $k^{a}(Y)$ in $\Omega$. Let $Y^{\prime}$ be the smooth projective $k^{a}$-curve corresponding to the compositum of $k^{a}(Y)$ and $k^{a}\left(X^{\prime}\right)$. Since the field 
extensions $k^{a}(Y)$ and $k^{a}\left(X^{\prime}\right)$ are linearly disjoint over $k^{a}(X)$, the cover $\psi: Y^{\prime} \rightarrow X$ has degree $p d$ :

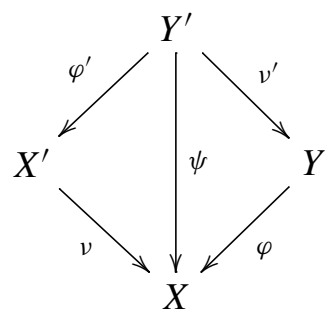

We prove this construction yields a morphism of stacks $\mathbb{F}: \mathbb{M}_{\varphi} \rightarrow \mathbb{M}_{\psi}$. Let $l \subset k^{a}$ be a finite extension of $k$. Set $X_{l}=X_{k} \times_{k} l$ and $X_{l}^{\prime}=X_{k}^{\prime} \times_{k} l$ and consider $\varphi_{l}: Y_{l} \rightarrow X_{l}$ an $l$-model of $\varphi$. In the construction above, one can replace $X, X^{\prime}, Y$ by $X_{l}, X_{l}^{\prime}, Y_{l}$. The $l$-curve $Y_{l}^{\prime}$ corresponding to the compositum of the two function fields $l\left(X_{l}^{\prime}\right)$ and $l\left(Y_{l}\right)$ is smooth, projective, geometrically integral (because $l$ is algebraically closed in the compositum) curve, and the $l$-cover $\psi_{l}: Y_{l}^{\prime} \rightarrow X_{l}$ is an $l$-model of $\psi$. We define the morphism $\mathbb{F}$ by setting $\mathbb{F}\left(\varphi_{l}\right)=\psi_{l}$. Since the function $f$ has been chosen in $k(X)$, the functor $\mathbb{F}$ maps cartesian squares to cartesian squares. Thus $\mathbb{F}$ is a morphism of stacks. According to Proposition 2.5, if $l$ is a field of definition of $\varphi$ then $l$ is a field of definition of $\psi$ and if $\varphi$ has $k$ as field of moduli then $\psi$ has $k$ as field of moduli.

To prove the converse, we construct a morphism the other way around using Proposition 2.7. Let $v^{\prime}$ denote the Galois cover $Y^{\prime} \rightarrow Y$. We need to show that the group $\operatorname{Aut}\left(v^{\prime}\right)$ is normal in $\operatorname{Aut}_{k}(\psi)$. Let $\Phi^{\prime} \in \operatorname{Aut}_{k}(\psi)$. It induces maps $\Phi: Y \rightarrow Y$ and $\Psi: X \rightarrow X$ such that the following diagram commutes:

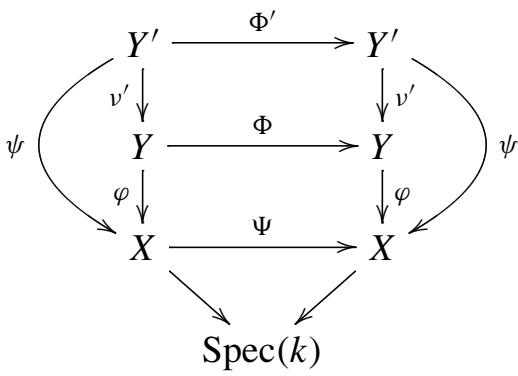

(horizontal arrows are morphisms of $k$-schemes). The existence of $\Psi$ results form $X$ being defined over $k$. The morphism $\Phi$ exists because $Y \stackrel{\varphi}{\rightarrow} X$ is the maximal subcover of $Y^{\prime} \stackrel{\psi}{\rightarrow} X$ unramified at the support of $f$. And $f$ is $k$-rational. Now if $\Lambda \in \operatorname{Aut}_{k^{a}}\left(v^{\prime}\right)$, that is, $v^{\prime} \circ \Lambda=v^{\prime}$, then

$$
v^{\prime} \circ \Phi^{\prime} \circ \Lambda=\Phi \circ v^{\prime} \circ \Lambda=\Phi \circ v^{\prime}=v^{\prime} \circ \Phi^{\prime}
$$


so $\Phi^{\prime} \circ \Lambda \circ \Phi^{\prime-1} \in \operatorname{Aut}_{k^{a}}\left(v^{\prime}\right)$, which was to be proved. So we have a morphism $\mathbb{G}$ : $\mathbb{M}_{\psi} \rightarrow \mathbb{M}_{\varphi}$ of stacks and the lemma follows.

Remark. The functor $\mathbb{E}: \mathbb{M}_{\varphi} \rightarrow \mathbb{M}_{\psi}$ is not fully faithful because $\psi$ has more automorphisms than $\varphi$. This is why we cannot apply point (3) of Proposition 2.5 here. We construct another functor $\mathbb{G}: \mathbb{M}_{\psi} \rightarrow \mathbb{M}_{\varphi}$ instead and we apply points (1) and (2) of Proposition 2.5 to either functors $\mathbb{F}$ and $\mathbb{G}$ successively. We notice that $\mathbb{G}$ is a left inverse of $\mathbb{F}$.

Next, we show that the base curve can be assumed to have genus greater than 2 .

Proposition 3.2. Let $X_{k}$ be a smooth, projective, geometrically integral curve over $k$ and set $X=X_{k} \times_{k} k^{a}$. Let $Y$ be a smooth projective and integral curve over $k^{a}$ and let $\varphi: Y \rightarrow X$ be a degree $d$ cover. There exist a smooth, projective, geometrically integral curve $X_{k}^{\prime}$ over $k$ of genus greater than 2 and a cover $\varphi^{\prime}: Y^{\prime} \rightarrow X_{k}^{\prime} \times_{k} k^{a}$ having the same field of moduli and the same fields of definition as $\varphi$.

Proof. We use the construction and notation of diagram (4) above. We further assume that the chosen function $f$ has degree at least 3. By the Hurwitz genus formula, the curve $X^{\prime}$ has a genus greater than or equal to 2 . This construction yields a morphism of stacks $\mathbb{F}: \mathbb{M}_{\varphi} \rightarrow \mathbb{M}_{\varphi^{\prime}}$. The cover $\varphi: Y \rightarrow X$ is the maximal subcover of $\psi: Y^{\prime} \rightarrow X$ unramified at the support of $f$. Therefore, there exists a morphism from $\operatorname{Aut}_{k^{a}}\left(\varphi^{\prime}\right) \rightarrow \operatorname{Aut}_{k^{a}}(\varphi)$. This morphism is bijective because $k^{a}\left(X^{\prime}\right)$ and $k^{a}(Y)$ are linearly disjoint over $k^{a}(X)$. So the morphism $\mathbb{E}$ is fully faithful. We conclude by invoking Proposition 2.5.

We now prove that one can assume that the base curve has no nontrivial $k^{a}$ automorphism.

Proposition 3.3. Let $X_{k}$ be a smooth, projective, geometrically integral curve over $k$ and set $X=X_{k} \times_{k} k^{a}$. Let $Y$ be a smooth projective and integral curve over $k^{a}$ and let $\varphi: Y \rightarrow X$ be a cover. There exists a smooth, projective, geometrically integral curve $X_{k}^{\prime}$ over $k$, of genus greater that 2 , such that $X^{\prime}=X_{k}^{\prime} \times_{k} k^{a}$ has no nontrivial automorphism and there exists a cover $\varphi^{\prime}: Y^{\prime} \rightarrow X^{\prime}$ having the same field of moduli and the same fields of definition as $\varphi$.

Proof. Thanks to Proposition 3.2, one can assume that the genus $g(X)$ of $X$ is greater than 2. So the group $\operatorname{Aut}(X)$ of $k^{a}$-automorphisms is finite. Let $p \geq 3$ be a prime integer.

We first show that there exists a nonconstant function $f \in k(X)$ that is nonsingular above $2,-2$ and $\infty$, of degree greater than $2+4 p(g(X)-1)+2 p^{2}$, such that the set $f^{-1}(\{-2,2\})$ is not invariant by any nontrivial automorphism of $X$, and such that the set of singular values of $\varphi$ does not meet the set $f^{-1}(\{2,-2, \infty\})$. Indeed, let $D$ be a simple effective divisor on $X$ with degree greater than $2+$ 
$4 p(g(X)-1)+2 p^{2}$. We also assume that $D$ is disjoint from the set of singular values of $\varphi$ and the linear space $L(D)$ associated with $D$ generates $k^{a}(X)$ over $k^{a}$. In particular, for every $\theta \in \operatorname{Aut}(X)$, this linear space is not contained in the Kernel of $\theta-\mathrm{Id}$. It is not contained in the kernel of $\theta+\mathrm{Id}$ either because it contains $k^{a}$. If $D$ has been chosen of large enough degree, the functions in $L(D)$ having degree less than the degree of $D$ are contained in a finite union of strict vector subspaces. Therefore there exists a nonconstant function $f \in L(D)$ such that $\operatorname{deg}(f)=\operatorname{deg}(D)$ and $\theta(f) \neq \pm f$ for all $\theta \in \operatorname{Aut}(X) \backslash\{\operatorname{Id}\}$. By construction, this function is not singular above $\infty$ and $f^{-1}(\infty)$ does not meet the singular values of $\varphi$. We can also assume that $f \in k\left(X_{k}\right)$.

By construction, the function $f^{2}$ has no nontrivial automorphism (in short, $\left.\operatorname{Aut}_{k^{a}\left(f^{2}\right)}\left(k^{a}(X)\right)=\{\operatorname{Id}\}\right)$. Using Lemma 7.3, we deduce that almost all the fibers of $f^{2}$ are nonsingular and not fixed by any nontrivial automorphism of $\operatorname{Aut}(X)$. In particular, there exists $\lambda \in k^{*}$ such that the fiber of $f^{2}$ above $\lambda^{2}$ is nonsingular, not fixed by any nontrivial automorphism in $\operatorname{Aut}(X)$ and does not meet the singular values of $\varphi$. The function $2 f / \lambda$ satisfies all the required properties. Denote it by $f$.

Now the equation $h^{p}+h^{-p}-f=0$ defines a regular extension of $k\left(X_{k}\right)$. Let $X_{k}^{\prime \prime}$ be the smooth, projective, geometrically integral curve associated with this function field. We denote by $w$ the automorphism of $X_{k}^{\prime \prime}$ given by $w(h)=h^{-1}$ and by $X_{k}^{\prime}$ the quotient $X_{k}^{\prime \prime} /\langle w\rangle$; this is a smooth, projective, geometrically integral $k$-curve, covering $X_{k}$ by a degree $p \quad k$-cover $v_{k}: X_{k}^{\prime} \rightarrow X_{k}$. Extending scalars to $k^{a}$, we obtain a Galois cover $X^{\prime \prime} \rightarrow X$ of $k^{a}$-curves, with Galois group $D_{p}$, and whose singular values are exactly $f^{-1}(\{2,-2, \infty\})$. Since the subgroup $\langle w\rangle$ is self-normalized in $D_{p}$, the quotient by this subgroup is a subcover $v: X^{\prime} \rightarrow X$ of $k^{a}$-curves of degree $p$ having no nontrivial automorphism.

Because the ramification loci do not meet, the function fields $k^{a}\left(X^{\prime \prime}\right)$ and $k^{a}(Y)$ are linearly disjoint over $k^{a}(X)$. Let $Y^{\prime}$ and $Y^{\prime \prime}$ be the smooth, projective, integral curves corresponding to the compositum of $k^{a}(Y)$ with $k^{a}\left(X^{\prime}\right)$ and $k^{a}\left(X^{\prime \prime}\right)$, respectively. We have the diagram

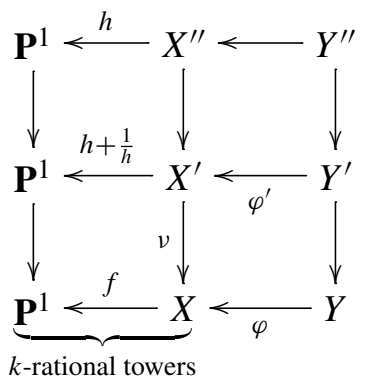

The cover $Y^{\prime \prime} \rightarrow Y$ is again a $D_{p}$-Galois cover and the cover $Y^{\prime \prime} \rightarrow Y^{\prime}$ has degree 2. Let us show that the cover $\varphi^{\prime}: Y^{\prime} \rightarrow X^{\prime}$ has the expected properties. 
First of all, it is clear that the construction above yields a morphism of stacks $\mathbb{F}: \mathbb{M}_{\varphi} \rightarrow \mathbb{M}_{\varphi^{\prime}}$. The Galois equivariance is a direct consequence of the fact that the middle tower is defined over $k$. This morphism is in fact fully faithful because the subcover $\varphi: Y \rightarrow X$ of $v \circ \varphi^{\prime}: Y^{\prime} \rightarrow X$ is the maximal subcover unramified at $f^{-1}(\{2,-2, \infty\})$.

It remains to prove that the curve $X^{\prime}$ has no nontrivial automorphism. Let $\theta^{\prime}$ be an automorphism of $X^{\prime}$. Call $Z$ the image of $v \times\left(v \circ \theta^{\prime}\right): X^{\prime} \rightarrow(X \times X)$. Let $\pi_{1}: X \times X \rightarrow X$ be the projection onto the first factor. The map $v$ factors as $v: X^{\prime} \rightarrow Z \stackrel{\pi_{1}}{\longrightarrow} X$ and it has prime degree $p$. So $Z$ is either isomorphic to $X$ or birationaly equivalent to $X^{\prime}$. In the latter case, the geometric genus of $Z$ would be $>\frac{1}{4} \operatorname{deg}(f) p \geq 1+2 p(g(X)-1)+p^{2}$ by the Hurwitz genus formula. But the bidegree of $Z$ is $\leq(p, p)$. So, by Lemma 7.1, its virtual arithmetic genus is less than $1+2 p(g(X)-1)+p^{2}$, a contradiction. Thus $Z$ is a correspondence of bidegree $(1,1)$ that defines an automorphism $\theta$ of $X$ such that $\theta \circ v=v \circ \theta^{\prime}$. Such an automorphism preserves the ramification data of $v$, that of its Galois closure $X^{\prime \prime} \rightarrow X$, and that of the unique subcover of degree 2 of the cover $X^{\prime \prime} \rightarrow X$. Since this last cover is exactly ramified above $f^{-1}(\{-2,2\})$, we deduce that $\theta=\mathrm{Id}$ and then that $\theta^{\prime}$ is a $k^{a}$-automorphism of the cover $v$. Since $v$ has no nontrivial automorphism, $\theta^{\prime}=$ Id.

\section{Quasiprojective surfaces}

In this section we prove the following theorem:

Theorem 4.1. Let $k$ be a field of characteristic zero. Let $X_{k}$ be a smooth, projective, geometrically integral curve over $k$ and set $X=X_{k} \times_{k} k^{a}$. Let $Y$ be a smooth, projective, integral curve over $k^{a}$ and let $\varphi: Y \rightarrow X$ be a nonconstant morphism of $k^{a}$ curves. Then there exists a smooth quasiprojective integral $k^{a}$-surface having the same field of moduli and the same fields of definition as $\varphi$.

First of all, by Propositions 3.2 and 3.3, one can assume the base curve $X$ to have genus greater than 2 and no nontrivial $k^{a}$-automorphism. Let $G(\varphi) \subset X \times Y$ be the graph of $\varphi$. Let $U$ be the open complementary set of $G(\varphi)$ in $X \times Y$. We show that the surface $U$ has all the expected properties. We call it the mark of the cover $\varphi: Y \rightarrow X$. We first prove two lemmas.

Lemma 4.2. Let $l / k$ be a finite extension of $k$ inside $k^{a}$. Let $X_{k}$ be a smooth, projective, geometrically integral $k$-curve. Set $X=X_{k} \times_{k} k^{a}$ and assume that the genus of $X$ is greater than 2 and that $X$ has no nontrivial $k^{a}$-automorphism. Let $U_{l}$ and $V_{l}$ be the marks of two nontrivial geometrically integral l-covers $\varphi_{l}: Y_{l} \rightarrow X_{l}$ and $\psi_{l}: Z_{l} \rightarrow X_{l}$, where $X_{l}=X_{k} \times_{k} l$.

Then every morphism of covers between $\varphi_{l}: Y_{l} \rightarrow X_{l}$ and $\psi_{l}: Z_{l} \rightarrow X_{l}$ induces a morphism between the corresponding marks $U_{l}$ and $V_{l}$. Conversely, every 
surjective l-morphism from $U_{l}$ to $V_{l}$ is equal to $\mathrm{Id} \times \gamma_{l}$, where $\gamma_{l}: Y_{l} \rightarrow Z_{l}$ is an $l$-morphism between the covers $\varphi_{l}: Y_{l} \rightarrow X_{l}$ and $\psi_{l}: Z_{l} \rightarrow X_{l}$.

Proof. An $l$-morphism between the covers $Y_{l} \stackrel{\varphi_{l}}{\longrightarrow} X_{l}$ and $Z_{l} \stackrel{\psi_{l}}{\longrightarrow} X_{l}$ is a morphism of $l$-curves $\gamma_{l}: Y_{l} \rightarrow Z_{l}$ such that $\psi_{l} \circ \gamma_{l}=\varphi_{l}$. The product $\operatorname{Id} \times \gamma_{l}: X_{l} \times Y_{l} \rightarrow X_{l} \times Z_{l}$ maps the graph of $\varphi_{l}$ to the graph of $\psi_{l}$ and also the mark $U_{l}$ to the mark $V_{l}$.

Conversely, let $v_{l}$ be a surjective $l$-morphism form $U_{l}$ to $V_{l}$. We denote by $v: U \rightarrow V, \varphi: Y \rightarrow X, \psi: Z \rightarrow X$ the base change to $k^{a}$ of $v_{l}, \varphi_{l}, \psi_{l}$, respectively. Let $y$ be a closed $k^{a}$-point of $Y$. Let $\pi_{2}: X \times Z \rightarrow Z$ be the projection onto the second factor. The restriction of $\pi_{2} \circ v$ to $(X \times\{y\}) \cap U$ is a constant because the genus of $X$ is less than that of $Z$. We denote by $\gamma(y)$ this constant; this defines a morphism $\gamma: Y \rightarrow Z$ which cannot be constant since $v$ is surjective. Let $\pi_{1}: X \times Z \rightarrow X$ be the projection onto the first factor. The restriction of $\pi_{1} \circ v$ to $(X \times\{y\}) \cap U$ is a morphism $\beta_{y}$ with values in $X$. Let $F \subset Y$ the set of closed $k^{a}$-points of $Y$ such that the morphism $\beta_{y}$ is constant. This is a closed set, and a finite one because $v$ is surjective. For a closed $k^{a}$-point $y \notin F$, the morphism $\beta_{y}$ induces an automorphism of $X$, which is trivial because $X$ has no nontrivial automorphism. Thus $v(x, y)=(x, \gamma(y))$ for every closed $k^{a}$-point $x$ on $X$ and $y$ on $Y$ with $y \notin F$ and $(x, y) \in U$. Let $x$ be a closed $k^{a}$-point of $X$. The restriction of $\pi_{1} \circ v$ to $(\{x\} \times Y) \cap U$ is constant and equal to $x$ on the nonempty open set $(\{x\} \times(Y-F)) \cap U$. So it is a constant function. So $F$ is empty and $v$ is the restriction of $\operatorname{Id} \times \gamma$ to $U$. Thus $\operatorname{Id} \times \gamma$ maps $U$ to $V$ and therefore $\psi \circ \gamma=\varphi$. Moreover $\gamma$ must be defined over $l$ since $v, U, V$ are defined over $l$.

Lemma 4.3. Suppose $X_{k}$ is a smooth, projective, geometrically integral k-curve. Assume that $X=X_{k} \times_{k} k^{a}$ has genus greater than 2 and no nontrivial $k^{a}$-automorphism. Let $U$ be the mark of a nonconstant $k^{a}$-cover $\varphi: Y \rightarrow X$, where $Y$ is a smooth, projective, integral $k^{a}$-curve. Then

(1) $k$ is the field of moduli of $U$ (in the category of quasiprojective varieties) if and only if it is the field of moduli of the cover $\varphi: Y \rightarrow X$, and

(2) an algebraic extension of $k$ is a field of definition of $U$ if and only if it is a field of definition of the cover $\varphi: Y \rightarrow X$.

Proof. It is clear that the construction of the mark from the cover commutes with base change. This yields a morphism of stacks $\mathbb{F}: \mathbb{M}_{\varphi} \rightarrow \mathbb{M}_{U}$, which is fully faithful according to Lemma 4.2. The result follows by Proposition 2.5. In particular, $\mathbb{F}$ has an inverse functor $\mathbb{G}: \mathbb{M}_{U} \rightarrow \mathbb{M}_{\varphi}$.

\section{Proper normal surfaces}

In this section we prove the following theorem: 
Theorem 5.1. Let $k$ be a field of characteristic zero. Let $X_{k}$ be a smooth, projective, geometrically integral curve over $k$ and set $X=X_{k} \times_{k} k^{a}$. Let $Y$ be a smooth projective, integral curve over $k^{a}$ and let $\varphi: Y \rightarrow X$ be a cover. Assume that $k$ is the field of moduli of $\varphi$. Then, there exists a proper, normal and integral surface $S$ over $k^{a}$, having $k$ as field of moduli, and having the same fields of definition as $\varphi$.

We construct the surface in question as a cover of the product $X \times Z$, strongly ramified along the graph of $\psi$, where $\psi: Z \rightarrow X$ is a well chosen cover derived from $\varphi$.

5a. Construction of the surface $S$. The construction of the surface is divided into several steps.

Step 1 (starting point). We keep notation and assumptions of Theorem 5.1. We denote by $g(X)$ the genus of $X$ and by $d$ the degree of the cover $\varphi$. According to Proposition 3.3, we may assume that $g(X)$ is at least 2 and that $X$ has no nontrivial automorphism over $k^{a}$.

Step 2 (a system of generators $f_{1}, \ldots, f_{I}$ of the function field $k\left(X_{k}\right)$ ). We need many $k$-rational functions on $X$.

Lemma 5.2 (the functions $f_{i}$ on $X$ and the primes $p_{i}$ ). There exist $I \in \mathbf{N}^{*}$, some prime integers $p_{1}, \ldots, p_{I}>d$, and functions $f_{1}, \ldots, f_{I} \in k\left(X_{k}\right)$ satisfying the following conditions:

(1) The functions $\left(f_{i}\right)_{1 \leq i \leq I}$ generate the field $k\left(X_{k}\right)$ over $k$.

(2) For every $1 \leq i \leq I$ and every $\lambda \in k^{a}$, none of the functions $f_{i}-\lambda$ is a $p_{i}$-th power in $k^{a}(X)$.

(3) Let $\Pi=\prod_{i=1}^{I} p_{i}$ and let $M$ and $m$ be the maximum and minimum, respectively, among the degrees of the $f_{i}$. Then

$$
1+2(g(X)-1) \Pi+\Pi^{2}<m \leq \operatorname{deg}\left(f_{i}\right) \leq M
$$

for all $i$ such that $1 \leq i \leq I$.

Proof. We first choose a finite generating system $\left(h_{j}\right)_{1 \leq j \leq J}$ of $k\left(X_{k}\right)$ over $k$. We assume that none of the $h_{j}$ is a power in $k^{a}(X)$. We set $I=2 J$ and let $\Pi=\prod_{i=1}^{I} p_{i}$ be the product of the first $I$ prime integers greater than the degree $d$ of $\varphi$. We choose two distinct prime integers $a$ and $b$, both greater than $1+2(g(X)-1) \Pi+\Pi^{2}$. For every $1 \leq j \leq J$, we set

$$
f_{j}=h_{j}^{a} \quad \text { and } \quad f_{j+J}=h_{j}^{b} .
$$

We can choose $a$ and $b$ in so that none of the functions $f_{i}-\lambda$ is a $p_{i}$-th power in $k^{a}(X)$ for $\lambda \in k^{a}$ and $1 \leq i \leq I$ : this is evident for $\lambda=0$. If $\lambda \neq 0$ and if $h_{i}^{a}-\lambda=\prod_{0 \leq k \leq a-1}\left(h_{i}-\zeta_{a}^{k} \lambda^{1 / a}\right)$ is a power, then $h_{i}$ has at least $a$ distinct singular 
values. This is impossible if we choose an $a$ greater than the number of singular values of $h_{i}$.

We also note that the $\left(f_{i}\right)_{1 \leq i \leq I}$ generate $k\left(X_{k}\right)$ over $k$ and that they all have a degree greater than $1+2(g(X)-1) \Pi+\Pi^{2}$, as expected.

Step 3 (a cover $\psi: Z \rightarrow X$ of large enough degree). Let $p$ be a prime integer greater than $(g(X)+I M) \Pi$. We call $Z$ the curve and $\psi: Z \rightarrow X$ the degree $p d$ cover given by Proposition 3.1. The genus of $Z$ is greater than $d p>(g(X)+I M) \Pi$ and the covers $\varphi$ and $\psi$ have the same field of moduli and the same fields of definition.

Step 4 (a system of functions $g_{1}, \ldots, g_{I}$ on $X \times Z$ ). Using the previous functions $f_{i}$, we define functions on $X \times Z$.

Lemma 5.3 (the functions $g_{i}$ on $X \times Z$ ). For every $1 \leq i \leq I$, let $g_{i}$ be the function on $X \times Z$ defined by

$$
g_{i}(P, Q)=f_{i}(\psi(Q))-f_{i}(P) .
$$

Then

(1) the negative part $\left(g_{i}\right)_{\infty}$ of the divisor of $g_{i}$ is $\left(f_{i}\right)_{\infty} \times Z+X \times\left(f_{i} \circ \psi\right)_{\infty}$,

(2) the positive parts $\left(g_{i}\right)_{0}$ are such that $\operatorname{gcd}_{i}\left(\left(g_{i}\right)_{0}\right)=G(\psi)$, where $G(\psi)$ is the graph of $\psi$, and

(3) for every point $P \in X$ the function $Q \mapsto g_{i}(P, Q)$ on $P \times Z$ is not a $p_{i}$-th power.

Proof. The first two points are easy. To prove the third one, we notice that each function $f_{i}$ is such that none of the $f_{i}-\lambda$ for $\lambda \in k^{a}$ is a $p_{i}$-th power (Lemma 5.2). Since the degree $p d$ of $\psi$ is prime to $p_{i}$, none of the function $f_{i} \circ \psi-\lambda$ is a $p_{i}$-th power in $k^{a}(Z)$. Condition 3 follows.

We stress that if $\psi$ is defined over a field $l$, then so are the functions $g_{i}$.

Step 5 (the surface $S)$. Let $k^{a}(X \times Z)$ be the field of functions of $X \times Z$. We define a regular radicial extension of $k^{a}(X \times Z)$ by setting

$$
y_{i}^{p_{i}}=g_{i} \quad \text { for every } 1 \leq i \leq I .
$$

We denote by $S$ the normalization of $X \times Z$ in the latter radicial extension. It is a normal surface by construction and there is a ramified cover

$$
\chi: S \rightarrow X \times Z
$$

that is Galois over $k^{a}$ with Galois group $\prod_{i=1}^{I} \mathbf{Z} / p_{i} \mathbf{Z}$. 
5b. The group of automorphisms of $S$. We denote by $A$ the group of $k^{a}$-automorphisms of $\psi$. An element in $A$ induces a $k^{a}$-automorphism of $X \times Z$, and this latter automorphism can be lifted uniquely to an automorphism of $k^{a}(S) / k^{a}$ that fixes all $y_{i}$ and stabilizes $k^{a}(X \times Z)$. In the sequel we shall use the same notation for an automorphism of $\psi$, the induced automorphism of $X \times Z$ and its lift to $S$. In other words, $A$ can be identified with a subgroup of $\operatorname{Aut}_{k^{a}}(S)$, the group of $k^{a}$-automorphisms of $S$.

Another subgroup of $\operatorname{Aut}_{k^{a}}(S)$ is the Galois group $B=\prod_{i=1}^{I} \mathbf{Z} / p_{i} \mathbf{Z}$ of the extension $k^{a}(S) / k^{a}(X \times Z)$. The groups $A$ and $B$ are the sets of $\alpha$ and $\beta$, respectively, such that the following diagrams commute:
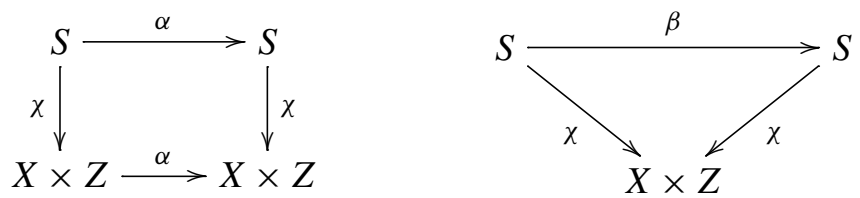

It is clear that $A \times B \subset \operatorname{Aut}_{k^{a}}(S)$. We now prove that this inclusion is an equality. To this end, we introduce a family of curves on $S$.

Lemma 5.4 (the curves $E_{Q}$ ). For any point $Q$ on $Z$, we call $E_{Q}$ the inverse image of $X \times Q$ by $\chi$ and we denote by $\chi_{Q}: E_{Q} \rightarrow X \times Q$ the restriction of $\chi$ to $E_{Q}$. The geometric genus of $E_{Q}$ can be bounded from above:

$$
g\left(E_{Q}\right) \leq(g(X)+I M) \Pi<g(Z),
$$

and the genus of any nontrivial subcover of $\chi_{Q}$ can be bounded from below:

$$
1+2(g(X)-1) \Pi+\Pi^{2}<m \leq g\left(\text { nontrivial subcover of } \chi_{Q}: E_{Q} \rightarrow X\right) .
$$

Proof. If $Q$ is the generic point on $Z$, then $E_{Q}$ is a geometrically integral curve and $\chi_{Q}$ is a degree $\Pi$, geometrically connected cover. The degree of the ramification divisor of this cover is bounded from above by the product $2 I M$ (where $I$ is the number of functions in the family $\left(f_{i}\right)_{i}$ and $M$ is the maximum of the degrees of these functions). The upper bound follows.

As for the lower bound, let us consider a nontrivial subcover of $\chi_{Q}$. Such a cover has degree at least $p_{1} \geq 3$ and its ramification divisor has degree at least $m$ (where $m$ is the minimum among the degrees of the functions $f_{i}$ ). So its genus is greater than $m$ and the lower bound follows.

Lemma 5.5. The group $\operatorname{Aut}(S)$ of $k^{a}$-automorphisms of $S$ is $A \times B$.

Proof. Let $\theta$ be a $k^{a}$-automorphism of $S$. Let $Q$ be the generic point of $Z$. We know from inequality (6) of Lemma 5.4 that $g\left(E_{Q}\right)<g(Z)$. We deduce that $\theta\left(E_{Q}\right)=E_{\alpha(Q)}$, where $\alpha$ is a $k^{a}$-automorphism of $Z$. 
We now prove that the isomorphism between $E_{Q}$ and $E_{\alpha(Q)}$ induced by $\theta$ makes the following diagram commute:

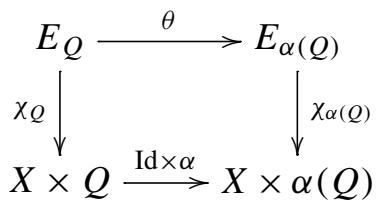

Indeed, the cartesian product of the maps $\chi_{Q}$ and $\chi_{\alpha(Q)} \circ \theta$ defines a morphism

$$
E_{Q} \stackrel{\chi_{Q} \times\left(\chi_{\alpha(Q)} \circ \theta\right)}{\longrightarrow} X \times X
$$

whose image $W$ is a divisor with bidegree $\leq(\Pi, \Pi)$. Using Lemma 7.1 we deduce that the arithmetic genus of $W$ is smaller than or equal to $1+2(g(X)-1) \Pi+\Pi^{2}$. Let $\pi_{1}: X \times X \rightarrow X$ be the projection on the first factor. The morphism $\chi_{Q}$ factors as

$$
\chi_{Q}: E_{Q} \rightarrow W \stackrel{\pi_{1}}{\longrightarrow} X .
$$

The map $W \stackrel{\pi_{1}}{\longrightarrow} X$ is a birational isomorphism, else it would define a nontrivial subcover of $\chi_{Q}: E_{Q} \rightarrow X$. But we know from inequality (7) of Lemma 5.4 that such a subcover has geometric genus greater than or equal to $m>1+2(g(X)-1) \Pi+\Pi^{2}$. A contradiction. We deduce that $W$ is a correspondence of bidegree $(1,1)$. Since $X$ has no nontrivial $k^{a}$-automorphism we deduce that diagram (8) commutes.

We now prove that $\alpha \in A$. We have just showed that $\theta$ induces an isomorphism between the covers $\chi_{Q}: E_{Q} \rightarrow X$ and $\chi_{\alpha(Q)}: E_{\alpha(Q)} \rightarrow X$. Therefore these two covers have the same ramification data: For every $1 \leq i \leq I$, the points $P$ such that $f_{i}(P)=f_{i}(\psi(Q))$ and those such that $f_{i}(P)=f_{i}(\psi(\alpha(Q)))$ are the same. Thus

$$
f_{i}(\psi(Q))=f_{i}(\psi(\alpha(Q))) \quad \text { for all } i
$$

therefore $\psi(Q)=\psi(\alpha(Q))$, because the $f_{i}$ generate $k^{a}(X)$ over $k^{a}$ (Lemma 5.2). So $\psi=\psi \circ \alpha$, and $\alpha \in A$.

Diagram (8) implies that the map $\chi_{\alpha(Q)} \circ \theta: E_{Q} \rightarrow E_{\alpha(Q)}$ is equal to $(\operatorname{Id} \times \alpha) \circ \chi_{Q}$. And this is $\chi_{\alpha(Q)} \circ \alpha$ according to diagram (5). We set $\beta=\theta \circ \alpha^{-1}$ and we check that $\chi_{\alpha(Q)} \circ \beta=\chi_{\alpha(Q)}$. Since $Q$ is generic and $\alpha$ surjective, we deduce that $\chi \circ \beta=\chi$ so $\beta \in B$. We conclude that $\theta=\beta \alpha \in A \times B$ as was to be shown.

Remark. We have proved something slightly stronger than Lemma 5.5: the group of birational $k^{a}$-automorphisms of $S$ is $A \times B$. We won't need this stronger result.

5c. Field of moduli and fields of definition of S. To prove Theorem 5.1, we have to show that the cover $\varphi$ and the surface $S$ share the same field of moduli and the same fields of definition. One can replace the cover $\varphi$ by the cover $\psi$, since those two covers have the same field of moduli and fields of definition. 
The construction of Section 5a yields a morphism of stacks $\mathbb{F}: \mathbb{M}_{\psi} \rightarrow \mathbb{M}_{S}$. To see that, let us consider an extension $l \subset k^{a}$ of $k$ and let $\psi_{l}: Z_{l} \rightarrow X_{l}$ be an $l$-model of $\psi$. We follow the lines of the construction above, replacing $\psi$ by $\psi_{l}$. Since the functions $f_{i}$ are $k$-rational, the functions $g_{i}$ lie in $l\left(X_{l} \times Z_{l}\right)$. Then the radical extension defined by the equations $y_{i}^{p_{i}}=g_{i}$ is a regular extension of $l\left(X_{l} \times Z_{l}\right)$. The normalization of $X_{l} \times Z_{l}$ in this extension is a surface $S_{l}$ which is defined over $l$. This surface $S_{l}$ is an $l$-model of $S$ and the morphism $\mathbb{F}$ is defined on objects by $\mathbb{F}\left(\psi_{l}\right)=S_{l}$. Because functions $f_{i}$ are $k$-rational, $\mathbb{F}$ is a morphism of stacks. According to Proposition 2.5, $k$ is the field of moduli of $S$ and every field of definition of $\psi($ or $\varphi)$ is a field of definition of $S$.

Unfortunately, $\mathbb{F}$ is not fully faithful. As we did in Proposition 3.1, we use Proposition 2.7 to construct a morphism the other way around. The group $\operatorname{Aut}_{k^{a}}(S)$ is a normal subgroup of $\operatorname{Aut}_{k}(S)$. The action of $\operatorname{Aut}_{k}(S)$ on $\operatorname{Aut}_{k^{a}}(S)$ stabilizes the unique subgroup of order $\Pi$, which is nothing but $B=\operatorname{Aut}_{k^{a}}(\chi)$. Let $U$ be the mark of the cover $\psi$. This is the complementary set of the branch locus of the quotient map $\chi: S \rightarrow X \times Z$. According to Proposition 2.7, taking the complementary set of the branch locus of a quotient map defines a morphism of stacks $\mathbb{G}: \mathbb{M}_{S} \rightarrow \mathbb{M}_{U}$. Therefore, every field of definition of $S$ is a field of definition of the mark of $\psi$ and then also a field of definition of $\psi$ by Lemma 4.3. Indeed the proof of this lemma provides a morphism from $\mathbb{M}_{U}$ to $\mathbb{M}_{\psi}$ and the proof of Proposition 3.1 provides a morphism from $\mathbb{M}_{\psi}$ to $\mathbb{M}_{\varphi}$.

\section{Curves}

In this section we prove Theorem 1.2. We shall make use of the surface $S$ constructed in Section 5, so we keep the notation there. We know that $S$ has field of moduli $k$ and the same fields of definition as the initial cover $\varphi: Y \rightarrow X$ (or equivalently $\psi: Z \rightarrow X$ ). The main idea is to draw on $S$ a singular (but stable) curve inheriting the field of moduli and fields of definition of $S$, and then to deform it to obtain a smooth projective curve.

6a. Two stable curves. In Section 5a, we have constructed a cover $\chi: S \rightarrow X \times Z$ strongly ramified along the graph of $\psi: Z \rightarrow X$. For any point $P$ on $X$, we call $F_{P}$ the inverse image of $P \times Z$ by $\chi$ and $\chi_{P}: F_{P} \rightarrow P \times Z$ the corestriction of $\chi$ to $P \times Z$. We call $\Gamma$ the union of the supports of all divisors of the functions $g_{i}$ of Lemma 5.3. It contains the ramification locus of the cover $\chi$.

Lemma 6.1. There exist two nonconstant $k$-rational functions $f, g \in k^{a}(X)$ such that

(1) the divisor $\left((f)_{0}+(f)_{\infty}\right) \times Z$ crosses $\Gamma$ transversally; 
(2) the divisor $X \times\left((g \circ \psi)_{0}+(g \circ \psi)_{\infty}\right)$ crosses $\Gamma \cup\left[\left((f)_{0}+(f)_{\infty}\right) \times Z\right]$ transversally;

(3) any $k^{a}$-automorphism of $Z$ that stabilizes the fiber $(g \circ \psi)_{0}$ is an automorphism of the cover $\psi$ (note that the preceding condition implies that this fiber is simple);

(4) for any zero $P$ of $f$, the cover $g \circ \psi \circ \chi_{P}: F_{P} \rightarrow \mathbf{P}^{1}$ has no automorphism other than the elements of $A \times B$. So $\operatorname{Aut}_{k^{a}}\left(g \circ \psi \circ \chi_{P}\right)=\operatorname{Aut}_{k^{a}}\left(\psi \circ \chi_{P}\right)=A \times B$.

Proof. Let $f \in k^{a}(X)$ be a $k$-rational nonconstant function. We apply Lemma 7.2 to $k, X, Z, \Gamma$ and $f$. We deduce that there exist two distinct scalars $x$ and $y$ in $k^{a}$ such that $(f)_{x} \times Z$ and $(f)_{y} \times Z$ cross transversally $\Gamma$. We can even choose $x$ and $y$ in $k$ and such that for every point $P$ in $f^{-1}(x)$ or $f^{-1}(y)$, the fiber of every function $f_{i} \in k(X)$ above $f_{i}(P)$ does not meet the singular values of $\psi$, that is,

$$
f_{i}^{-1}\left(f_{i}(P)\right) \cap\{\text { singular values of } \psi\}=\varnothing \quad \text { for all } P \in f^{-1}(x) \cup f^{-1}(y) .
$$

We replace $f$ by $(f-x) /(f-y)$ and the first condition is fulfilled.

Now, for every zero $P$ of $f$, we see that $F_{P}$ is smooth and geometrically integral, because $(f)_{0} \times Z$ crosses transversally the ramification locus $\Gamma$ of $\chi$. We now prove that

$$
\operatorname{Aut}_{k^{a}}\left(\psi \circ \chi_{P}\right)=A \times B .
$$

Indeed the function field $k^{a}\left(F_{P}\right)$ is the compositum

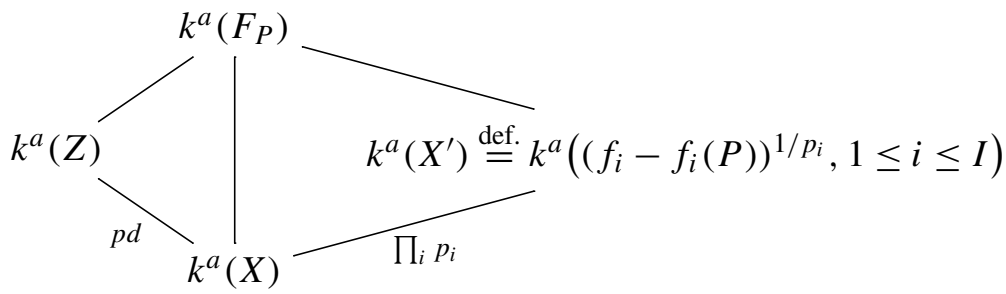

where $X^{\prime} \rightarrow X$ is an abelian cover with Galois group $B=\prod_{i=1}^{I} \mathbf{Z} / p_{i} \mathbf{Z}$. The $k^{a}(X)$ extensions $k^{a}(Z)$ and $k^{a}\left(X^{\prime}\right)$ are linearly disjoint (their degrees are coprime and one of them is Galois) and condition (9) implies that the extension $k^{a}(Z) / k^{a}(X)$ is not ramified above the zeros of the functions $f_{i}-f_{i}(P)$.

Now, any subcover of $X^{\prime} \rightarrow X$ is ramified above the zeros of at least one of the functions $f_{i}-f_{i}(P)$. The same is true for any subcover of $F_{P} \rightarrow Z$. We deduce that $Z \rightarrow X$ is the maximal subcover of $F_{P} \rightarrow X$ that is not ramified above the zeros of the functions $f_{i}-f_{i}(P)$. Therefore any $k^{a}(X)$-automorphism of $k^{a}\left(F_{P}\right)$ stabilizes $k^{a}(Z)$. Thus

$$
\operatorname{Aut}_{k^{a}(X)}\left(k^{a}\left(F_{P}\right)\right)=\operatorname{Aut}_{k^{a}(X)}\left(k^{a}(Z)\right) \times \operatorname{Aut}_{k^{a}(X)}\left(k^{a}\left(X^{\prime}\right)\right),
$$


as was to be shown.

Next we look for a function $g$ in $k(X)$ such that $g \circ \psi$ has no $k^{a}$-automorphism but elements of $A$ and, for every zero $P$ of $f$, the cover $g \circ \psi \circ \chi_{P}$ has no $k^{a}-$ automorphism but elements of $\operatorname{Aut}_{k^{a}}\left(\psi \circ \chi_{P}\right)=A \times B$. According to Lemma 7.4, the functions in $k(X)$ that do not fulfill all these conditions lie in a finite union of strict sub- $k$-algebras. Therefore there exists such a function $g$.

According to Lemma 7.2, the scalars $x$ in $k$ such that $(g \circ \psi)_{x}$ does not cross $\Gamma \cup\left[\left((f)_{0}+(f)_{\infty}\right) \times Z\right]$ transversally are finitely many. According to Lemma 7.3, the $x$ in $k$ such that $(g \circ \psi)_{x}$ has a $k^{a}$-automorphism not in $A$ are finitely many. Therefore there exist two distinct scalars $x$ and $y$ in $k$ such that $(g \circ \psi)_{x}$ and $(g \circ \psi)_{y}$ cross $\Gamma \cup\left[\left((f)_{0}+(f)_{\infty}\right) \times Z\right]$ transversally and $(g \circ \psi)_{x}$ has no automorphism but those in $A$. We replace $g$ by $(g-x) /(g-y)$ and the last three conditions are satisfied.

The curves $C_{0}$ and $D_{0}$. Let $C_{0}$ be the curve on $X \times Z$ with equation

$$
f(P) \times g \circ \psi(Q)=0 .
$$

Let $D_{0}$ be the inverse image of $C_{0}$ by $\chi$. These are singular curves over $k^{a}$. The two following lemmas are concerned with the stability and the automorphism groups of these two curves.

Lemma 6.2. The curve $C_{0}$ is stable and $\operatorname{Aut}_{k^{a}}\left(C_{0}\right) \simeq A$.

Proof. The curve $C_{0}$ is geometrically reduced because the zeros of $f$ and $g \circ \psi$ are simple. The singular points on $C_{0}$ are the couples $(P, Q)$ on $X \times Z$ such that $f(P)=g \circ \psi(Q)=0$. These are ordinary double points. Therefore $C_{0}$ is semistable. It is geometrically connected also. Its irreducible components are isomorphic to $X$ or $Z$. So they all have genus $\geq 2$. Therefore $C_{0}$ is a stable curve.

We now prove that the group of $k^{a}$-automorphisms of $C_{0}$ is the group $A$ of $k^{a}$ automorphisms of $\psi$. It is clear that $A$ is included in $\operatorname{Aut}_{k^{a}}\left(C_{0}\right)$. Conversely, let $\theta$ be a $k^{a}$-automorphism of $C_{0}$. Then $\theta$ permutes the irreducible components of $C_{0}$. Some of these components are isomorphic to $X$, and the others are isomorphic to $Z$. Since $X$ and $Z$ are not $k^{a}$-isomorphic, $\theta$ stabilizes the two subsets of components. If we restrict $\theta$ to a component isomorphic to $X$ and then compose with the projection on $X$, we obtain a nonconstant $k^{a}$-morphism from $X$ to itself. This morphism must be the identity because $X$ has no nontrivial $k^{a}$-automorphism. Therefore $\theta$ stabilizes each component isomorphic to $Z$. The singular points on such a component are the zeros of $g \circ \psi$. The set of these zeros is stabilized by no $k^{a}$-automorphism of $Z$ but those of $\psi$ by (3) of Lemma 6.1. So the restriction of $\theta$ to any component isomorphic to $Z$ is in $A$. Composing $\theta$ with a well chosen element in $A$, we may assume that $\theta$ is trivial on one component isomorphic to $Z$. Therefore $\theta$ stabilizes every component isomorphic to $X$. Since these components 
have no nontrivial automorphism, $\theta$ acts trivially on them. Now let $P \times Z$ be a component of $C_{0}$ isomorphic to $Z$. The restriction of $\theta$ to it is an automorphism that fixes the singular points. These points are the zeros of $g \circ \psi$. So the restriction of $\theta$ to $P \times Z$ is in $A$. Since $A$ acts faithfully on the set of zeros of $g \circ \psi$, we deduce that $\theta$ acts trivially on every component isomorphic to $Z$.

Examining the full group of $k^{a}$-automorphisms of $D_{0}$ seems difficult to us. We restrict our attention to the subgroup $\operatorname{Aut}_{k^{a}}^{\text {adm. }}\left(D_{0}\right)$ of admissible automorphisms. We first explain what we mean by an admissible automorphism.

We first note that the components of $D_{0}$ are of two different kinds. Some of them are covers of some $X \times Q$ where $Q$ is a zero of $g \circ \psi$. We denote such a component by $E_{Q}$. The other components are covers of some $P \times Z$ where $P$ is a $k^{a}$-zero of $f$. Such a component is denoted by $F_{P}$. We call $\chi_{P}: F_{P} \rightarrow P \times Z$ and $\chi_{Q}: E_{Q} \rightarrow X \times Q$ the restrictions of $\chi$ to components of $D_{0}$. Now let $T$ be a singular point on $D_{0}$ such that $\chi(T)=(P, Q)$. So $T$ lies in the intersection of $E_{Q}$ and $F_{P}$. The point on $E_{Q}$ corresponding to $T$ is denoted $U$. The point on $F_{P}$ corresponding to $T$ is denoted $V$. So $\chi_{Q}(U)=P$ and $\chi_{P}(V)=Q$. Condition (2) in Lemma 6.1 implies that $f \circ \chi_{Q}$ is a uniformizing parameter for $E_{Q}$ at $U$; likewise, condition (1) implies that $g \circ \psi \circ \chi_{P}$ is a uniformizing parameter for $E_{Q}$ at $F_{P}$ at $V$. Let $\theta$ be an automorphism of $D_{0}$ and let $T^{\prime}=\left(U^{\prime}, V^{\prime}\right)$ be the image of $T=(U, V)$ by $\theta$. We write $\chi\left(T^{\prime}\right)=\left(P^{\prime}, Q^{\prime}\right)$. We observe that $f \circ \chi_{Q^{\prime}} \circ \theta$ is a uniformizing parameter for $E_{Q}$ at $U$ and $g \circ \psi \circ \chi_{P^{\prime}} \circ \theta$ is a uniformizing parameter for $F_{P}$ at $V$.

We say that $\theta$ is an admissible automorphism of $D_{0}$ if for every singular point $T$ of $D_{0}$, we have

$$
\frac{f \circ \chi_{Q^{\prime}} \circ \theta}{f \circ \chi_{Q}}(U) \times \frac{g \circ \psi \circ \chi_{P^{\prime}} \circ \theta}{g \circ \psi \circ \chi_{P}}(V)=1,
$$

where $\chi(T)=(P, Q)$ and $\chi(\theta(T))=\left(P^{\prime}, Q^{\prime}\right)$. The justification for this definition is given in Section 6b. Admissible automorphisms form a subgroup of the group of $k^{a}$-automorphisms of $D_{0}$.

Lemma 6.3. The curve $D_{0}$ is stable and $\operatorname{Aut}_{k^{a}}^{\mathrm{adm}}\left(D_{0}\right) \simeq A \times B$.

Proof. It is clear that $A \times B$ acts faithfully on $D_{0}$, and the corresponding automorphisms are admissible.

We can now prove that the curve $D_{0} \subset S$ is a stable curve. Points (1) and (2) of Lemma 6.1 imply that the ramification locus $\Gamma$ of $\chi$ does not contain any singular points of $C_{0}$. Therefore every singular point on $C_{0}$ gives rise to $\operatorname{deg}(\chi)$ singular points on $D_{0}$. These are all ordinary double points. To prove that $D_{0}$ is connected, we observe that the function $g_{i}$ restricted to any irreducible component of $C_{0}$ is not a $p_{i}$-th power because none of the functions $f_{i}-\lambda, \lambda \in k^{a}$ is a $p_{i}$-th power 
(and the $f_{i} \circ \psi-\lambda$ are not either) as shown in Lemma 5.2. Also the irreducible components of $D_{0}$ correspond bijectively to those of $C_{0}$.

Now let us prove that $\operatorname{Aut}_{k^{a}}^{\text {adm. }}\left(D_{0}\right) \simeq A \times B$. The components $F_{P}$ and $E_{Q}$ have different genera. Therefore any $k^{a}$-automorphism $\theta$ of $D_{0}$ stabilizes the set of all components $F_{P}$ (and also the set of all $E_{Q}$ ).

Let $Q$ and $Q^{\prime}$ be two $k^{a}$-zeros of $g \circ \psi$ such that $\theta\left(E_{Q}\right)=E_{Q^{\prime}}$. As in the proof of Lemma 5.5, we notice that the image of $E_{Q}$ in the product $X \times X$ by the morphism $\chi_{Q} \times \chi_{Q^{\prime}} \circ \theta$ has an arithmetic genus no more than $1+2(g(X)-1) \Pi+\Pi^{2}$. Again, this implies that this image is $k^{a}$-isomorphic to $X$ (otherwise, this image would have geometric genus bigger than $1+2(g(X)-1) \Pi+\Pi^{2}$ by the Hurwitz formula). Since $X$ has no $k^{a}$-automorphism, we deduce that $\theta$ induces an isomorphism of covers between the restrictions $\chi_{Q}: E_{Q} \rightarrow X$ and $\chi_{Q^{\prime}}: E_{Q^{\prime}} \rightarrow X$ of $\chi$. Thus

$$
\chi_{Q}=\chi_{Q^{\prime}} \circ \theta \text {. }
$$

This implies that $\theta$ stabilizes every component $F_{P}$, where $P$ is any $k^{a}$-zero of $f$. Indeed, let $T=(U, V) \in E_{Q} \cap F_{P}$ be a singular point, where $P$ is a $k^{a}$-zero of $f$ and $Q$ is a $k^{a}$-zero of $g \circ \psi$. We have $\chi(T)=(P, Q) \in X \times Z$ and there exist $P^{\prime} \in X\left(k^{a}\right)$ and $Q^{\prime} \in Z\left(k^{a}\right)$ such that $\theta(T) \in F_{P^{\prime}} \cap E_{Q^{\prime}}$. We deduce from Equation (11) that

$$
P^{\prime}=\chi_{Q^{\prime}} \circ \theta(T)=\chi_{Q}(T)=P .
$$

We conclude that $P=P^{\prime}$ and $\theta\left(F_{P}\right)=F_{P}$.

Now, we deduce from formulas (10) and (11) that

$$
\frac{g \circ \psi \circ \chi_{P} \circ \theta}{g \circ \psi \circ \chi_{P}}(V)=1 \text {. }
$$

Denote by $\theta_{P}$ the restriction of $\theta$ to $F_{P}$. This is an automorphism of $F_{P}$. We prove $\theta_{P}$ is the restriction to $F_{P}$ of an element of $A \times B$. To this end, we introduce the function $h_{P}=g \circ \psi \circ \chi_{P} \in k^{a}\left(F_{P}\right)$. The degree of $h_{P}$ is $\operatorname{deg}(g) \times p d \times \Pi$ and its zeros are all simple. These zeros are the intersection points between $F_{P}$ and the other components of $D_{0}$. Since $\theta_{P}$ permutes these zeros, the functions $h_{P} \circ \theta_{P}$ and $h_{P}$ have the same divisor of zeros. Therefore the only possible poles of the function $h_{P} /\left(h_{P} \circ \theta_{P}\right)-1$ are the poles of $h_{P}$, and its degree is $\leq h_{P}$. But according to (12), the zeros of $h_{P}$ are also zeros of $h_{P} /\left(h_{P} \circ \theta_{P}\right)-1$. So if the function $h_{P} /\left(h_{P} \circ \theta_{P}\right)-1$ is nonzero, it has the same divisor as $h_{P}$. Therefore there exists a constant $c \in k^{a}$ such that

$$
\frac{h_{P}}{h_{P} \circ \theta_{P}}-1=c h_{P} \quad \text { or equivalently } \quad \frac{1}{h_{P} \circ \theta_{P}}=\frac{1}{h_{P}}+c .
$$

Since $\theta_{P}$ has finite order $e$ and $k^{a}$ has characteristic zero, we deduce that $c e=0$, and then $c=0$. Then $h_{P} \circ \theta_{P}=h_{P}$ and $\theta_{P}$ is an automorphism of the cover 
$h_{P}=g \circ \psi \circ \chi_{P}: F_{P} \rightarrow \mathbf{P}^{1}$. According to point (4) of Lemma 6.1, we deduce that $\theta_{P}$ is the restriction to $F_{P}$ of an element in $A \times B$. We replace $\theta$ by $\theta$ composed with the inverse of this element. So we can now assume that $\theta$ acts trivially on $F_{P}$ for some $P$. In particular $\theta$ fixes every singular point on $F_{P}$. So $\theta$ stabilizes every component $E_{Q}$. The restriction $\theta_{Q}$ of $\theta$ to $E_{Q}$ is an automorphism of $\chi_{Q}$ according to (11). Further $\theta_{Q}$ fixes a point (and every point) in the unramified fiber above $P$ of the Galois cover $\chi_{Q}: E_{Q} \rightarrow X$. Therefore $\theta_{Q}$ is the identity. We have proved that $\theta$ is trivial on every component $E_{Q}$.

To conclude, we prove that $\theta$ is also trivial on the components $F_{P^{\prime}}$ for every zero $P^{\prime}$ of $f$. Let us recall that we have already assumed this to be true for one of these zeros. We call $\theta_{P^{\prime}}$ the restriction of $\theta$ to $F_{P^{\prime}}$. We have already proved that $\theta_{P^{\prime}}$ is the restriction of an element in $A \times B$. Further $\theta_{P^{\prime}}$ fixes all the singular points of $D_{0}$ lying on $F_{P^{\prime}}$. These points are the zeros of $g \circ \psi \circ \chi_{P^{\prime}}$. Therefore, it only remains to be proved that the action of $A \times B$ on the set of zeros of $g \circ \psi \circ \chi_{P^{\prime}}$ is free. This is the case for elements in $B$ because the zeros of $g \circ \psi$ are, by hypothesis, unramified in the Galois cover $\chi_{P^{\prime}}: F_{P^{\prime}} \rightarrow Z$. This is also true for elements in $A \times B$ because the action of $A$ on the set of zeros of $g \circ \psi$ is free.

6b. Deformations. We now deform the two stable curves $C_{0}$ and $D_{0}$. If $t \in k^{a}$ is a scalar, we call $C_{t} \subset W=X \times Z$ the curve with equation $f(P) \times g(\psi(Q))=t$. We call $D_{t}$ the inverse image of $C_{t}$ by $\chi$. In this and the next paragraph, we prove that for almost all scalars $t$ in $k$, the curve $D_{t}$ is smooth, geometrically integral, with $k^{a}$-automorphism group equal to $A \times B$, and with the same field of moduli and the same fields of definition as the original cover $\varphi$. To this end, we would like to consider the families $\left(C_{t}\right)_{t}$ and $\left(D_{t}\right)_{t}$ as fibrations above $\mathbf{P}^{1}$. We should be careful however: the family $\left(C_{t}\right)_{t}$ has base points. So we blow up $W=X \times Z$ along

$$
\Delta=\left((f)_{\infty} \times(g \circ \psi)_{0}\right) \cup\left((f)_{0} \times(g \circ \psi)_{\infty}\right) .
$$

Note that $\Delta$ is the union of $2 \times \operatorname{deg}(f) \times \operatorname{deg}(g \circ \psi)$ simple geometric points. We call $W_{\infty, \infty} \subset W=X \times Z$ the complementary open set of

$$
\left((f)_{\infty} \times Z\right) \cup\left(X \times(g \circ \psi)_{\infty}\right) \quad \text { in } X \times Z .
$$

We similarly define $W_{0,0}, W_{0, \infty}, W_{\infty, 0}$. These four open sets cover $X \times Z$.

Let $\mathbf{P}^{1}=\operatorname{Proj}\left(k^{a}\left[T_{0}, T_{1}\right]\right)$ be the projective line over $k^{a}$. We set $F=1 / f$ and $G=1 / g$. Let $C_{\infty, 0} \subset W_{\infty, 0} \times \mathbf{P}^{1}$ be the set of $\left(P, Q,\left[T_{0}: T_{1}\right]\right)$ such that $f(P) T_{0}=$ $G(\psi(Q)) T_{1}$. Let $C_{0, \infty} \subset W_{0, \infty} \times \mathbf{P}^{1}$ be the set of $\left(P, Q,\left[T_{0}: T_{1}\right]\right)$ such that $g(\psi(Q)) T_{0}=F(P) T_{1}$. Let $C_{\infty, \infty} \subset W_{\infty, \infty} \times \mathbf{P}^{1}$ be the set of $\left(P, Q,\left[T_{0}: T_{1}\right]\right)$ such that $f(P) g(\psi(Q)) T_{0}=T_{1}$. Let $C_{0,0} \subset W_{0,0} \times \mathbf{P}^{1}$ be the set of $\left(P, Q,\left[T_{0}: T_{1}\right]\right)$ such that $T_{0}=F(P) G(\psi(Q)) T_{1}$. We glue together these four algebraic varieties and obtain a variety $C \subset W \times \mathbf{P}^{1}$. Let $\pi_{W}: C \rightarrow W$ be the projection on the 
first factor and let $\pi_{C}: C \rightarrow \mathbf{P}^{1}$ be the projection on $\mathbf{P}^{1}$. This is a flat, projective, surjective morphism.

Let $D \subset S \times \mathbf{P}^{1}$ be the inverse image of $C$ by $\chi \times$ Id where Id : $\mathbf{P}^{1} \rightarrow \mathbf{P}^{1}$ is the identity. This is the blow up of $S$ along $\chi^{-1}(\Delta)$. Note that $\chi^{-1}(\Delta)$ is the union of $\operatorname{deg}(\chi) \times \operatorname{deg}(f) \times \operatorname{deg}(g \circ \psi)$ simple geometrical points because $\chi$ is unramified above $\Delta$. Actually, $D$ is the normalization of $C$ in $k^{a}\left(S \times \mathbf{P}^{1}\right)$. We denote by $\chi: D \rightarrow C$ the corresponding morphism. We call $\pi_{S}: D \rightarrow S$ the projection on the first factor. We call $\pi_{D}: D \rightarrow \mathbf{P}^{1}$ the projection on the second factor. This is the composed morphism $\pi_{D}=\pi_{C} \circ \chi$. This is a flat, proper and surjective morphism.

Let $\mathbf{A}^{1} \subset \mathbf{P}^{1}$ be the spectrum of $k^{a}[T]$, where $T=T_{1} / T_{0}$. Using the function $T$ we identify $\mathbf{P}^{1}\left(k^{a}\right)$ and $k^{a} \cup\{\infty\}$. If $t$ is a point on $\mathbf{P}^{1}\left(k^{a}\right)$ we denote by $C_{t}$ the fiber of $\pi_{C}$ above $t$ and by $D_{t}$ the fiber of $\pi_{D}$ above $t$. The restriction of $\pi_{W}$ to $C_{t}$ is a closed immersion. So we can see $C_{t}$ as a curve on $W=X \times Z$. Similarly, the restriction of $\pi_{S}$ to $D_{t}$ is a closed immersion. So we can see $D_{t}$ as a curve drawn on $S$. In particular, the fiber of $\pi_{C}$ at 0 is isomorphic by $\pi_{W}$ to the stable curve $C_{0}$ introduced in Section 6a. Similarly, the fiber of $\pi_{D}$ at 0 is isomorphic by $\pi_{S}$ to the stable curve $D_{0}$ introduced in Section 6a.

Let us call $C_{\eta}$ the generic fiber of $\pi_{C}$ and $D_{\eta}$ the generic fiber of $\pi_{D}$.

We prove that the curve $C_{\eta}$ over $k^{a}\left(\mathbf{P}^{1}\right)$ is geometrically connected and that for almost every $t \in \mathbf{P}^{1}\left(k^{a}\right)$, the curve $C_{t}$ over $k^{a}$ is connected. According to Stein's factorization theorem [Liu 2002, Chapter 5, Exercise 3.11], we can factor $\pi_{C}: C \rightarrow \mathbf{P}^{1}$ as $\pi_{f} \circ \pi_{c}$, where $\pi_{c}$ has geometrically connected fibers and $\pi_{f}$ is finite and dominant. The fiber of $\pi_{f}$ above 0 is trivial because $C_{0}$ is connected and reduced. Therefore the degree of $\pi_{f}$ is 1 according to [Liu 2002, Chapter 5, Exercise 1.25]. Therefore $\pi_{f}$ is an isomorphism above a nonempty open set of $\mathbf{P}^{1}$. The generic fiber $C_{\eta}$ is geometrically connected over $k^{a}\left(\mathbf{P}^{1}\right)$ and for almost all $t \in \mathbf{P}^{1}\left(k^{a}\right)$ the curve $C_{t}$ over $k^{a}$ is connected.

We now prove that $C_{\eta}$ is smooth (and therefore geometrically integral). Indeed, it is smooth outside the points $(P, Q) \in C_{\eta} \subset X \times Z$, where $d f(P)=0$ and $d(g \circ \psi)(Q)=0$. Such points are defined over $k^{a}$. Therefore $f(P) \times g(\psi(Q))$ cannot take the transcendental value $T$ at these points.

The ramification locus $\Gamma \subset W$ of $\chi$ cuts the fiber $C_{0}$ transversally. Therefore it cuts the generic fiber $C_{\eta}$ transversally. So $D_{\eta}$ is smooth and geometrically integral. Thus for almost every $t \in k^{a}$ the fibers $C_{t}$ and $D_{t}$ are smooth and integral.

We now compute $\operatorname{Aut}_{k\left(\mathbf{P}^{1}\right)^{s}}\left(D_{\eta}\right)$. Let $R=k^{a} \llbracket T \rrbracket$ be the completed local ring at the point $T=0$ of $\mathbf{P}^{1}$. The curve $\hat{D}=D \times \mathbf{P}^{1} \operatorname{Spec}(R)$ is stable over $\operatorname{Spec}(R)$. According to [Liu 2002, Chapter 10, Proposition 3.38, Remark 3.39] the functor "automorphism group" $t \mapsto \operatorname{Aut}_{t}\left(\hat{D}_{t}\right)$ is representable by a finite unramified scheme over Spec $R$ and the specialization morphism $\operatorname{Aut}_{k^{a}((T))}\left(\hat{D}_{\eta}\right) \rightarrow \operatorname{Aut}_{k^{a}}\left(D_{0}\right)$ is injective. According to Lemma 7.6, the image of this morphism is included in the 
subgroup of admissible $k^{a}$-automorphisms of $D_{0}$. Since $\operatorname{Spec} R$ has no unramified cover, we can deduce

$$
A \times B \subset \operatorname{Aut}_{k^{a}\left(\mathbf{P}^{1}\right)^{s}}\left(D_{\eta}\right) \subset \operatorname{Aut}_{k^{a}((T))}\left(\hat{D}_{\eta}\right) \subset \operatorname{Aut}_{k^{a}}^{\mathrm{adm} .}\left(D_{0}\right) .
$$

We know that the rightmost group is equal to $A \times B$. So

$$
\operatorname{Aut}_{k^{a}}\left(\mathbf{P}^{1}\right)^{s}\left(D_{\eta}\right)=A \times B
$$

as was intended to be proved.

6c. Fields of moduli and fields of definition of fibers. We just have proved that for almost all $t \in \mathbf{A}^{1}(k)$, the fiber $D_{t}$ is smooth and geometrically integral. Using Lemma 7.7 on the specialization of the automorphism group we deduce that for almost all $t \in \mathbf{A}^{1}(k)$, the group of $k^{a}$-automorphisms of the fiber $D_{t}$ is isomorphic to the group of $k\left(\mathbf{A}^{1}\right)^{s}$-automorphisms of the generic fiber. Since the latter group is isomorphic to the automorphism group $A \times B$ of the surface $S$, we deduce that, for almost all $t$, the restriction map is an isomorphism:

$$
\operatorname{Aut}_{k^{a}}(S) \stackrel{\simeq}{\longrightarrow} \operatorname{Aut}_{k^{a}}\left(D_{t}\right) .
$$

Now let $t \in k$ be such that $D_{t}$ is smooth and geometrically integral and such that $\operatorname{Aut}_{k^{a}}\left(D_{t}\right)=A \times B$. We call $\pi_{t}: D_{t} \rightarrow S$ the corresponding embedding. We construct a functor $\mathbb{F}_{t}: \mathbb{M}_{S} \rightarrow \mathbb{M}_{\pi_{t}}$. We first define the image of an object by $\mathbb{F}_{t}$. Let $l \subset k^{a}$ be a finite extension of $k$ and $S_{l}$ an $l$-model of $S$. Using the functor $\mathbb{M}_{S} \rightarrow \mathbb{M}_{U}$ given in Section $5 \mathrm{c}$ followed by the functor $\mathbb{M}_{U} \rightarrow \mathbb{M}_{\psi}$ in the proof of Lemma 4.3, we obtain an $l$ model $\psi_{l}: Z_{l} \rightarrow X_{l}$ of the cover $\psi$, where $X_{l}=X_{k} \times{ }_{k} l$ and $Z_{l}$ is an $l$-model of $Z$. There is also an abelian cover $\chi_{l}: S_{l} \rightarrow X_{l} \times Z_{l}$ which is well defined up to an automorphism of $S_{l}$. We denote by $C_{t, l}$ the curve on $X_{l} \times Z_{l}$ with the equation $f \cdot g \circ \psi_{l}-t=0$. Let $D_{t, l}$ be the inverse image of $C_{t, l}$ by $\chi_{l}$. Let $\pi_{t, l}: D_{t, l} \hookrightarrow S_{l}$ be the inclusion map. The image of the object $S_{l}$ by the functor $\mathbb{F}_{t}$ is defined to be $\pi_{t, l}$. We still need to define the image of a morphism by the functor $\mathbb{F}_{t}$. Let $l^{\prime}$ be another finite extension of $k$ and let $\sigma: l \rightarrow l^{\prime}$ be a $k$-homomorphism. Let $S_{l^{\prime}}^{\prime}$ be an $l^{\prime}$-model of $S$ and let $\alpha: S_{l} \rightarrow S_{l^{\prime}}^{\prime}$ be a morphism above $\operatorname{Spec}(\sigma)$. We call $\pi_{t, l^{\prime}}^{\prime}: D_{t, l^{\prime}}^{\prime} \hookrightarrow S_{l^{\prime}}^{\prime}$ the image by $\mathbb{F}_{t}$ of $S_{l^{\prime}}^{\prime}$. Then $\alpha$ maps $D_{t, l}$ to $D_{t, l^{\prime}}^{\prime}$. We denote by $\beta$ the restriction of $\alpha$ to $D_{t, l}$. The image of $\alpha$ by $\mathbb{F}_{t}$ is defined to be the morphism $(\alpha, \beta)$ from $\pi_{t, l}$ to $\pi_{t, l^{\prime}}^{\prime}$. If we compose $\mathbb{F}_{t}: \mathbb{M}_{S} \rightarrow \mathbb{M}_{\pi_{t}}$ with the forgetful functor $\mathbb{M}_{\pi_{t}} \rightarrow \mathbb{M}_{D_{t}}$, we obtain a cartesian functor $\mathbb{G}_{t}: \mathbb{M}_{S} \rightarrow \mathbb{M}_{D_{t}}$. Further, identity (13) implies that the functor $\mathbb{G}_{t}$ is fully faithful. So, by Proposition 2.5, both $S$ and $D_{t}$ have $k$ as field of moduli and they have the same fields of definition. In view of Section $5 \mathrm{c}, D_{t}, \psi$ and $\varphi$ also share the same fields of definition. Theorem 1.2 is proved. 


\section{Six lemmas about curves and surfaces}

In this section we state and prove six lemmas that are needed in the proof of Theorem 1.2.

\section{7a. About curves and products of two curves.}

Lemma 7.1. Let $k$ be a algebraically closed field. Let $X$ and $Y$ be two projective, smooth and integral curves over $k$. Let $\beta$ be the genus of $X$ and let $\gamma$ be the genus of $Y$. We fix a geometric point $P$ on $X$ and a geometric point $Q$ on $Y$. We identify the curves $X$ and $X \times Q$ and the curves $Y$ and $P \times Y$. Let $D$ be a divisor on $X \times Y$ of bidegree $(b, c)$, that is, $b=X \cdot D$ and $c=Y \cdot D$. The virtual arithmetic genus $\pi$ of $D$ is at most $1+b c+c(\beta-1)+b(\gamma-1)$. When $b=c$ this bound reads $1+2 b(\beta-1)+b^{2}$.

Proof. The algebraic equivalence class of the canonical divisor on $X \times Y$ is $K=2(\beta-1) Y+2(\gamma-1) X$. The virtual arithmetic genus $\pi$ of $D$, as defined in [Hartshorne 1977, Exercise V-1.3], is $\pi=\frac{1}{2} D \cdot(D+K)+1$. Thus $\pi=D \cdot(D+2(\beta-1) Y+2(\gamma-1) X) / 2+1$. We deduce from Castelnuovo's and Severi's inequality (see [Hartshorne 1977, Exercise V-1.9]) that $D \cdot D \leq 2 b c$. This finishes the proof of the lemma.

Lemma 7.2. Let $k$ be an algebraically closed field. Let $X$ and $Y$ be two projective, smooth, integral curves over $k$. Let $D$ be an effective divisor without multiplicity on the surface $X \times Y$. Let $f \in k(X)$ be a nonconstant function. For all but finitely many scalars $x$ in $k$, the divisor $(f)_{x} \times Y$ crosses $D$ transversally, where $(f)_{x}$ is the positive part of the divisor of $f-x$.

Proof. We call $p_{X}: X \times Y \rightarrow X$ the projection on the first factor. Let $E$ be the set of points in $X(k)$ such that at least one of the following condition holds: $p_{X}^{-1}(P)$ contains a singular point on $D$, or $p_{X}^{-1}(P)$ contains a ramified point of the morphism $p_{X}: D \rightarrow X$, or the fiber $p_{X}^{-1}(P)$ is contained in $D$. The set $E$ is finite. For all $x \in k$ but finitely many, the fiber $f^{-1}(x)$ avoids $E$ and is simple.

Lemma 7.3. Let $k$ be an algebraically closed field. Let $X$ be a projective, smooth, integral curve over $k$ with genus at least 2. Let $f \in k(X)$ be a nonconstant function. We denote by $G$ the group of $k$-automorphisms of $f$. This is the set of all k-automorphisms $\theta$ of $X$ such that $f \circ \theta=f$. For any $x \in \mathbf{P}^{1}(k)$, we write $(f)_{x}=f^{-1}(x)$ for the fiber above $x$, and $G_{x}$ for the group of $k$-automorphisms of $X$ that stabilize the set of $k$-points of $(f)_{x}$. For all $x$ in $\mathbf{P}^{1}(k)$ but finitely many, we have $G_{x}=G$.

Proof. The group $H=\operatorname{Aut}_{k}(X)$ of $k$-automorphisms of $X$ is finite. Let $\theta$ be an automorphism in $H \backslash G$ and let $x \in \mathbf{P}^{1}(k)$. Assume that the $k$-points in $(f)_{x}$ are permuted by $\theta$. Let $P$ be one of them. Then $f \circ \theta(P)=f(P)=x$. So $P$ is a 
zero of the nonzero function $f \circ \theta-f$. For each $\theta$ there are finitely many such zeros. And the $\theta$ are finitely many. So the images by $f$ of such $P$ are finitely many also.

Lemma 7.4. Let $k$ be a field. Let $X_{k}$ be a projective, smooth, geometrically integral curve over $k$. Set $X=X_{k} \times_{k} k^{a}$ and assume that $X$ has genus at least 2 . Let $Y$ be a projective, smooth, integral curve over $k^{a}$ and let $\varphi: Y \rightarrow X$ be a nonconstant $k^{a}$-cover. If $f$ is any nonconstant function in $k^{a}(X)$, then $\operatorname{Aut}(\varphi) \subset \operatorname{Aut}(f \circ \varphi)$. Let $V \subset k\left(X_{k}\right)$ be the set of functions $f \in k\left(X_{k}\right)$ such that $\operatorname{Aut}(\varphi) \neq \operatorname{Aut}(f \circ \varphi)$. This set $V$ is contained in a finite union of strict $k$-subalgebras of $k\left(X_{k}\right)$.

Proof. The statement concerns the three function fields $k^{a}(f) \subset k^{a}(X) \subset k^{a}(Y)$, and the groups involved are the following ones:

$$
\left\{\begin{array}{l}
\operatorname{Aut}(\varphi)=\operatorname{Aut}_{k^{a}(X)}\left(k^{a}(Y)\right), \\
\operatorname{Aut}(f \circ \varphi)=\operatorname{Aut}_{k^{a}(f)}\left(k^{a}(Y)\right), \quad \Rightarrow \quad \operatorname{Aut}(\varphi) \subset \operatorname{Aut}(f \circ \varphi) \subset \operatorname{Aut}(Y) . \\
\operatorname{Aut}(Y)=\operatorname{Aut}_{k^{a}}\left(k^{a}(Y)\right),
\end{array}\right.
$$

Now, the set $V$ can be described as

$$
V=\left(\bigcup_{\theta \in \operatorname{Aut}(Y) \backslash \operatorname{Aut}(\varphi)} k^{a}(Y)^{\theta} \cap k^{a}(X)\right) \cap k\left(X_{k}\right)=\bigcup_{\theta \in \operatorname{Aut}(Y) \backslash \operatorname{Aut}(\varphi)} k^{a}(Y)^{\theta} \cap k\left(X_{k}\right) .
$$

This is a union of sets indexed by elements in the finite set $\operatorname{Aut}(Y) \backslash \operatorname{Aut}(\varphi)$ (recall that $\operatorname{Aut}(Y)$ is finite because the genus of $Y$ is at least 2). Since $\theta \notin \operatorname{Aut}(\varphi)$, each $k^{a}(Y)^{\theta} \cap k^{a}(X)$ is a strict subfield of $k^{a}(X)$ containing $k^{a}$. Therefore $k^{a}(Y)^{\theta} \cap$ $k\left(X_{k}\right) \subsetneq k\left(X_{k}\right)$.

7b. Deformation of an automorphism of a nodal curve. In this subsection we give a necessary condition for an automorphism of a nodal curve to extend to a given deformation of this curve. Let $R$ be a complete discrete valuation ring. Let $\pi$ be a uniformizing parameter and let $k$ be the residue field. Assume that $k$ is algebraically closed. Let $D$ be a semistable curve over $\operatorname{Spec}(R)$. Let $D_{\eta}$ be the generic fiber and $D_{0}$ the special fiber. Assume $D_{\eta}$ is smooth over the fraction field of $R$. Let $T$ be a singular point of $D_{0}$. According to [Liu 2002, Chapter 10, Corollary 3.22], the completion of the local ring of $D$ at $T$ takes the form

$$
\hat{\mathrm{O}}_{D, T}=R \llbracket f, g \rrbracket /\left\langle f g-\pi^{e}\right\rangle,
$$

where $e$ is a positive integer. This integer is called the thickness of $D$ at $T$. We also say that $f$ and $g$ form a coordinate system for $D$ at $T$. If we reduce modulo $\pi$, we obtain the completion of the local ring of $D_{0}$ at $T$ :

$$
\hat{\mathrm{O}}_{D_{0}, T}=\hat{\mathrm{O}}_{D, T} /\langle\pi\rangle=k \llbracket \bar{f}, \bar{g} \rrbracket /\langle\bar{f} \bar{g}\rangle,
$$


where $\bar{f}=f \bmod \pi$ and $\bar{g}=g \bmod \pi$. Because $T$ is an ordinary double point, $D_{0}$ has two branches $F$ and $G$ at $T$. These correspond to the two irreducible components of the completion at $T$. Be careful that these two branches may lie on the same irreducible component of $D_{0}$. Anyway, the functions $\bar{f}$ and $\bar{g}$ are the uniformizing parameters of either branches. We call $P$ and $Q$ the points of $F$ and $G$ above $T$.

Now let $T^{\prime}$ be another singular point of $D_{0}$, and let $f^{\prime}, g^{\prime}, e^{\prime}, F^{\prime}$, and $G^{\prime}$ be the corresponding data. Let $\theta$ be an automorphism of $D$ over $R$ such that $\theta(T)=T^{\prime}$ and $\theta(F)=F^{\prime}, \theta(G)=G^{\prime}$. The functions $f^{\prime} \circ \theta$ and $g^{\prime} \circ \theta$ form a coordinate system for $D$ at $T$. So $e^{\prime}=e$ and both $f^{\prime} \circ \theta / f$ and $g^{\prime} \circ \theta / g$ are units in $\hat{O}_{D, T}$ (indeed, in either fraction, the numerator and denominator have the same Weil divisor). Since $f \times g=\pi^{e}=f^{\prime} \circ \theta \times g^{\prime} \circ \theta$, we have $\frac{f^{\prime} \circ \theta}{f}(T) \times \frac{g^{\prime} \circ \theta}{g}(T)=1$. We reduce this identity modulo $\pi$ and obtain the following identity, where the first factor is a function on $F$ evaluated at $P$ and the second is a function on $G$ evaluated at $Q$ :

$$
\frac{\bar{f}^{\prime} \circ \bar{\theta}}{\bar{f}}(P) \times \frac{\bar{g}^{\prime} \circ \bar{\theta}}{\bar{g}}(Q)=1 .
$$

Definition 7.5. Let $R$ be a complete discrete valuation ring. Assume that the residue field $k$ is algebraically closed. Let $D$ be a semistable curve over $\operatorname{Spec}(R)$. The generic fiber of $D$ is assumed to be smooth. Assume we are given a coordinate system at each singular point of the special fiber $D_{0}$. Let $\bar{\theta}$ be an automorphism of the special fiber $D_{0}$. We say that $\bar{\theta}$ is admissible in $D / \operatorname{Spec}(R)$ if for every singular point $T$ of $D_{0}$, the image $\bar{\theta}(T)$ has the same thickness as $T$ in $D$, and if equality (14) holds true.

We have just proved this:

Lemma 7.6. With the notation of Definition 7.5, the set of automorphisms of $D_{0}$ that are admissible in $D / \operatorname{Spec}(R)$ is a subgroup of $\operatorname{Aut}_{k}\left(D_{0}\right)$. If $\theta$ is an automorphism of $D$ over $\operatorname{Spec}(R)$, its reduction $\bar{\theta}=\theta \bmod \pi$ is an automorphism of $D_{0}$ and is admissible in $D / \operatorname{Spec}(R)$.

One may compare this statement with [Wewers 1999, Theorem 3.1.1], where the deformation of morphisms between two distinct curves is studied.

Remark. The converse of Lemma 7.6 is not true. For example, consider the elliptic curve $E$ with modular invariant $j=0$ (or 1728). Every automorphism of $E$ is admissible because there are no singular points on the curve (the condition in Definition 7.5 is empty). However, the only automorphisms that can be extended to the generic elliptic curve are the identity and the involution.

7c. Automorphisms of curves in a family. We state and prove a standard lemma about specialization of automorphism groups. 
Lemma 7.7. Let $k$ be a field and let $U$ be a smooth, geometrically integral curve over $k$. Let $X$ be a quasiprojective, smooth, geometrically integral surface over $k$. Let $\pi: X \rightarrow U$ be a surjective, projective, smooth morphism of relative dimension 1 . Assume that for any point $x$ of $U$, the fiber $X_{x}$ at $x$ is geometrically integral. We call $\eta$ the generic point of $U$ and call $\bar{X}_{\eta}=X_{\eta} \times \operatorname{Spec}(k(U)) \operatorname{Spec}\left(k(U)^{a}\right)$ the generic fiber, seen as a curve over the algebraic closure of the function field of the basis $U$. We assume the genus of $X_{\eta}$ is at least 2.

There exists a nonempty open subset $V$ of $U$ over $k$ such that for any geometric point $x \in V\left(k^{a}\right)$ the group of $k^{a}$-automorphisms of the fiber at $x$ is equal to the group $\operatorname{Aut}_{k(U)}\left(\bar{X}_{\eta}\right)$ of automorphisms of $\bar{X}_{\eta}$.

The following proof was communicated to us by Qing Liu.

Proof. This is a consequence of a general result by Deligne and Mumford. Let $X \rightarrow S$ be a flat projective morphism over a noetherian scheme $S$. The functor $T \rightarrow \operatorname{Aut}_{T}\left(X_{T}\right)$ from the category of $S$-schemes to the category of groups is representable by a group scheme Aut $_{X / S}$ over $S$. See [Kollár 1996, Exercise 1.10.2] for example. When $X \rightarrow S$ is a stable curve with genus at least 2, Deligne and Mumford [1969, Theorem 1.11] prove that the scheme Aut $X / S$ is finite and unramified over $S$. In our lemma, $S$ is a smooth, geometrically integral curve $U$ over $k$. Replacing $S$ by a nonempty open subset, we may assume that $\operatorname{Aut}_{X / S}$ is finite étale over $S$. At the expense of a finite surjective base change $T \rightarrow S$, we may assume that the generic fiber of $\mathrm{Aut}_{X / S} \rightarrow S$ consists of rational points. So Aut $_{X / S} \rightarrow S$ is now a disjoint union of étale sections and the fibers have constant degree. In particular, the fibers are constant and the specialization maps $\operatorname{Aut}_{S}(X)=\operatorname{Aut}_{X / S}(S) \rightarrow \operatorname{Aut}_{S}\left(X_{S}\right)=\operatorname{Aut}_{X / S}(k(s))$ are isomorphisms.

\section{References}

[Coombes and Harbater 1985] K. Coombes and D. Harbater, "Hurwitz families and arithmetic Galois groups", Duke Math. J. 52:4 (1985), 821-839. MR 87g:14012 Zbl 0601.14023

[Couveignes and Granboulan 1994] J.-M. Couveignes and L. Granboulan, "Dessins from a geometric point of view", pp. 79-113 in The Grothendieck theory of dessins d'enfants (Luminy, 1993), edited by L. Schneps, London Math. Soc. Lecture Note Ser. 200, Cambridge Univ. Press, 1994. MR 96b:14015 Zbl 0835.14010

[Couveignes and Ros 2004] J.-M. Couveignes and N. Ros, "Des obstructions globales à la descente des revêtements”, Acta Arith. 114:4 (2004), 331-348. MR 2006h:11075 Zbl 1056.12002

[Dèbes and Emsalem 1999] P. Dèbes and M. Emsalem, "On fields of moduli of curves", J. Algebra 211:1 (1999), 42-56. MR 99k:14044 Zbl 0934.14019

[Dèbes and Fried 1994] P. Dèbes and M. D. Fried, "Nonrigid constructions in Galois theory", Pacific J. Math. 163:1 (1994), 81-122. MR 95c:12008 Zbl 0788.12001

[Deligne and Mumford 1969] P. Deligne and D. Mumford, "The irreducibility of the space of curves of given genus", Inst. Hautes Études Sci. Publ. Math. 36 (1969), 75-109. MR 41 \#6850 Zbl 0181.48803 
[Giraud 1964] J. Giraud, Méthode de la descente, Bull. Soc. Math. France Mém. 2, 1964. MR 32 \#7556

[Hartshorne 1977] R. Hartshorne, Algebraic geometry, Graduate Texts in Mathematics 52, Springer, New York, 1977. MR 57 \#3116 Zbl 0367.14001

[Kollár 1996] J. Kollár, Rational curves on algebraic varieties, Ergebnisse der Mathematik und ihrer Grenzgebiete (3) 32, Springer, Berlin, 1996. MR 98c:14001 Zbl 0877.14012

[Liu 2002] Q. Liu, Algebraic geometry and arithmetic curves, Oxford Graduate Texts in Mathematics 6, Oxford University Press, 2002. MR 2003g:14001 Zbl 0996.14005

[Mestre 1991] J.-F. Mestre, "Construction de courbes de genre 2 à partir de leurs modules”, pp. 313-334 in Effective methods in algebraic geometry (Castiglioncello, 1990), edited by T. Mora and

C. Traverso, Progr. Math. 94, Birkhäuser, Boston, MA, 1991. MR 92g:14022 Zbl 0752.14027

[Serre 1959] J.-P. Serre, Groupes algébriques et corps de classes, 2nd ed., Publications de l'institut de mathématique de l'université de Nancago 7, Hermann, Paris, 1959. MR 21 \#1973 Zbl 0097. 35604

[Silverman 1995] J. H. Silverman, "The field of definition for dynamical systems on $\mathbf{P}^{1}$ ", Compositio Math. 98:3 (1995), 269-304. MR 96j:11090 Zbl 0849.11090

[Vistoli 2005] A. Vistoli, "Grothendieck topologies, fibered categories and descent theory", pp. 1104 in Fundamental algebraic geometry, Math. Surveys Monogr. 123, Amer. Math. Soc., Providence, RI, 2005. MR 2223406

[Weil 1956] A. Weil, "The field of definition of a variety", Amer. J. Math. 78 (1956), 509-524. MR 18,601a

[Wewers 1999] S. Wewers, "Deformation of tame admissible covers of curves", pp. 239-282 in Aspects of Galois theory (Gainesville, FL, 1996), edited by H. Völklein et al., London Math. Soc. Lecture Note Ser. 256, Cambridge Univ. Press, 1999. MR 2001b:14048 Zbl 0995.14008

Communicated by Bjorn Poonen

Received 2009-09-11 Revised 2010-11-22 Accepted 2011-02-19

jean-marc.couveignes@math.u-bordeaux1.fr

Institut de Mathématiques de Bordeaux,

Université Bordeaux I et CNRS, 161, cours de la Libération, F-33405 Talence, France

http://www.math.u-bordeaux1.fr/ couveign/

hallouin@univ-tlse2.fr Département de mathématiques et informatique,

Université de Toulouse le Mirail, 5, allées Antonio Machado, F-31058 Toulouse, France

http://www.math.univ-toulouse.fr/ hallouin/ 


\section{Algebra \& Number Theory}

www.jant.org

\section{EDITORS}

MANAGING EDITOR

Bjorn Poonen

Massachusetts Institute of Technology

Cambridge, USA

\author{
EDITORIAL BOARD CHAIR \\ David Eisenbud \\ University of California \\ Berkeley, USA
}

BOARD OF EDITORS

Georgia Benkart

Dave Benson

Richard E. Borcherds

John H. Coates

J-L. Colliot-Thélène

Brian D. Conrad

Hélène Esnault

Hubert Flenner

Edward Frenkel

Andrew Granville

Joseph Gubeladze

Ehud Hrushovski

Craig Huneke

Mikhail Kapranov

Yujiro Kawamata

János Kollár

Yuri Manin

Barry Mazur

Philippe Michel

Susan Montgomery
University of Wisconsin, Madison, USA

University of Aberdeen, Scotland

University of California, Berkeley, USA

University of Cambridge, UK

CNRS, Université Paris-Sud, France

University of Michigan, USA

Universität Duisburg-Essen, Germany

Ruhr-Universität, Germany

University of California, Berkeley, USA

Université de Montréal, Canada

San Francisco State University, USA

Hebrew University, Israel

University of Kansas, USA

Yale University, USA

University of Tokyo, Japan

Princeton University, USA

Northwestern University, USA

Harvard University, USA

École Polytechnique Fédérale de Lausanne

University of Southern California, USA
Shigefumi Mori

Raman Parimala

Jonathan Pila

Victor Reiner

Karl Rubin

Peter Sarnak

Joseph H. Silverman

Michael Singer

Ronald Solomon

Vasudevan Srinivas

J. Toby Stafford

Bernd Sturmfels

Richard Taylor

Ravi Vakil

Michel van den Bergh

Marie-France Vignéras

Kei-Ichi Watanabe

Andrei Zelevinsky

Efim Zelmanov
RIMS, Kyoto University, Japan

Emory University, USA

University of Oxford, UK

University of Minnesota, USA

University of California, Irvine, USA

Princeton University, USA

Brown University, USA

North Carolina State University, USA

Ohio State University, USA

Tata Inst. of Fund. Research, India

University of Michigan, USA

University of California, Berkeley, USA

Harvard University, USA

Stanford University, USA

Hasselt University, Belgium

Université Paris VII, France

Nihon University, Japan

Northeastern University, USA

University of California, San Diego, USA

\section{PRODUCTION}

contact@msp.org

Silvio Levy, Scientific Editor

See inside back cover or www.jant.org for submission instructions.

The subscription price for 2011 is US $\$ 150 /$ year for the electronic version, and \$210/year (+ \$35 shipping outside the US) for print and electronic. Subscriptions, requests for back issues from the last three years and changes of subscribers address should be sent to Mathematical Sciences Publishers, Department of Mathematics, University of California, Berkeley, CA 94720-3840, USA.

Algebra \& Number Theory (ISSN 1937-0652) at Mathematical Sciences Publishers, Department of Mathematics, University of California, Berkeley, CA 94720-3840 is published continuously online. Periodical rate postage paid at Berkeley, CA 94704, and additional mailing offices.

ANT peer review and production are managed by EditFLOW ${ }^{\circledR}$ from Mathematical Sciences Publishers.

PUBLISHED BY

mathematical sciences publishers

http://msp.org/

A NON-PROFIT CORPORATION

Typeset in LATEX $_{\mathrm{E}}$

Copyright $\odot 2011$ by Mathematical Sciences Publishers 


\section{Algebra \& Number Theory}

Volume $5 \quad$ No. $4 \quad 2011$

Global descent obstructions for varieties

JeAn-Marc Couveignes and Emmanuel Hallouin

Specializations of elliptic surfaces, and divisibility in the Mordell-Weil group

PATRICK INGRAM

Explicit CM theory for level 2-structures on abelian surfaces

REINIER BRÖKER, DAVID GRUENEWALD and KRISTIN LAUTER

THOMAS BRÜSTLE and JIE ZHANG 